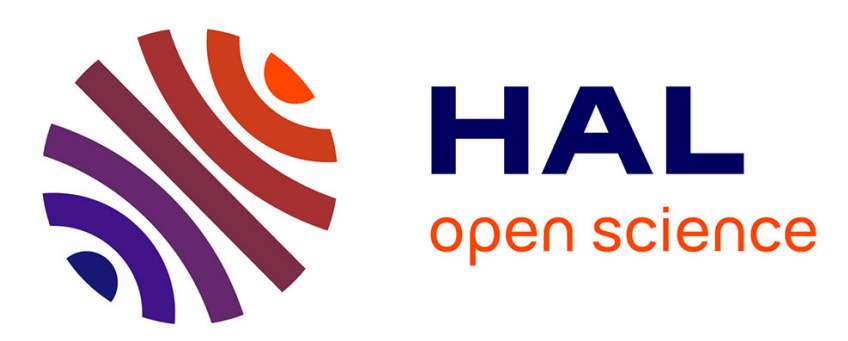

\title{
Boundary conditions for a one-sided numerical model of evaporative instabilities in sessile drops of ethanol on heated substrates
}

Sergey Semenov, Florian Carle, Marc Medale, David Brutin

\section{To cite this version:}

Sergey Semenov, Florian Carle, Marc Medale, David Brutin. Boundary conditions for a one-sided numerical model of evaporative instabilities in sessile drops of ethanol on heated substrates. Physical Review E , 2017, 96 (6), pp.063113. 10.1103/PhysRevE.96.063113 . hal-01769983

\section{HAL Id: hal-01769983 \\ https://hal.science/hal-01769983}

Submitted on 18 Apr 2018

HAL is a multi-disciplinary open access archive for the deposit and dissemination of scientific research documents, whether they are published or not. The documents may come from teaching and research institutions in France or abroad, or from public or private research centers.
L'archive ouverte pluridisciplinaire HAL, est destinée au dépôt et à la diffusion de documents scientifiques de niveau recherche, publiés ou non, émanant des établissements d'enseignement et de recherche français ou étrangers, des laboratoires publics ou privés. 


\title{
Boundary conditions for a one-sided numerical model of evaporative instabilities in sessile drops of ethanol on heated substrates
}

\author{
Sergey Semenov, ${ }^{1,2, *}$ Florian Carle, ${ }^{1,3}$ Marc Medale, ${ }^{1}$ and David Brutin ${ }^{1,4, \dagger}$ \\ ${ }^{1}$ Aix-Marseille University, IUSTI UMR 7343 CNRS, 13453 Marseille, France \\ ${ }^{2}$ Aix-Marseille University, MADIREL UMR 7246 CNRS, 13013 Marseille, France \\ ${ }^{3}$ Yale Quantum Institute, Yale University, New Haven, Connecticut 06511, USA \\ ${ }^{4}$ Institut Universitaire de France, 75231 Paris, France
}

(Received 5 July 2017; revised manuscript received 28 October 2017; published 19 December 2017)

\begin{abstract}
The work is focused on obtaining boundary conditions for a one-sided numerical model of thermoconvective instabilities in evaporating pinned sessile droplets of ethanol on heated substrates. In the one-sided model, appropriate boundary conditions for heat and mass transfer equations are required at the droplet surface. Such boundary conditions are obtained in the present work based on a derived semiempirical theoretical formula for the total droplet's evaporation rate, and on a two-parametric nonisothermal approximation of the local evaporation flux. The main purpose of these boundary conditions is to be applied in future three-dimensional (3D) one-sided numerical models in order to save a lot of computational time and resources by solving equations only in the droplet domain. Two parameters, needed for the nonisothermal approximation of the local evaporation flux, are obtained by fitting computational results of a 2D two-sided numerical model. Such model is validated here against parabolic flight experiments and the theoretical value of the total evaporation rate. This study combines theoretical, experimental, and computational approaches in convective evaporation of sessile droplets. The influence of the gravity level on evaporation rate and contributions of different mechanisms of vapor transport (diffusion, Stefan flow, natural convection) are shown. The qualitative difference (in terms of developing thermoconvective instabilities) between steady-state and unsteady numerical approaches is demonstrated.
\end{abstract}

DOI: 10.1103/PhysRevE.96.063113

\section{INTRODUCTION}

Evaporating sessile drops of simple and complex fluids are widely encountered in nature and have a great diversity of actual and potential applications. They include heat exchangers [1], patterns of nanoparticles deposition from evaporation of sessile drops (coffee-ring effect) [2,3], spraying of herbicides and pesticides on hydrophobic leaves [4], inkjet printing [5,6], microlens manufacturing [7], biological tissue engineering [8], and other biomedical applications (e.g., blood analysis $[9,10]$ and surfactant replacement therapy [11]). Due to a wide range of applications, sessile drops have been the subject of extensive experimental and theoretical studies, and still remain an interesting problem from both scientific and industrial points of view.

The physics of evaporation comprises many different processes, such as diffusive and convective (buoyancy and Stefan flow) vapor transport, the kinetics of vapor molecules transfer across the liquid-gas interface (Hertz-Knudsen-Langmuir equation), evaporative interface cooling, Marangoni flows, and instabilities. There are also complications associated with the contact line physics: slip at the solid-liquid interface and adsorbed precursor films (disjoining pressure action).

Despite of all these complicated physics involved in droplets evaporation, simplified isothermal and nonisothermal models of diffusion limited evaporation are widely used for the validation of experimental data. This is partly due to their simplicity and availability along with their qualitative ability to predict the droplet's evaporation rate. In this paper,

\footnotetext{
*s.semionov_1266@mail.ru

†david.brutin@univ-amu.fr
}

for the purpose of quantitative validation, we present a semiempirical theoretical model, which predicts an unsteady diffusion-limited evaporation rate for a nonisothermal pinned sessile droplet, taking into account Stefan flow in gas.

Our current research is focused on thermal Marangoni instabilities in sessile ethanol droplets, which develop spontaneously during evaporation. One distinctive type of these thermocapillary instabilities is called hydrothermal waves (HTWs). Conventional HTWs are observed in thin liquid layers whose surface is subject to a lateral temperature gradient [12-16]. In sessile droplets, however, the HTWs are driven by the process of evaporation which generates these temperature gradients naturally. These instabilities have been observed in droplets of volatile liquids (ethanol, methanol, FC-72) on heated substrates by few researchers [17-21].

Several studies, employing numerical methods to analyze unsteady evaporation of sessile drops, have been found in the literature. Among them, Barash et al. [22] used the finite difference method (FDM) and "a boundary interpolation in a vicinity of the drop surface"; Maki and Kumar [23] used lubrication approximation and the kinetic model of evaporation ("transferrate-limited evaporation") including the contribution of both capillarity and disjoining pressure on the liquid surface; Korenchenko and Beskachko [24] studied drop oscillations using the FDM; Yang et al. [25] studied evaporating water drops using the finite element method (FEM) and the arbitrary Lagrangian-Eulerian (ALE) formulation. An unsteady LatticeBoltzmann method (LBM) was used to study evaporating drops on patterned substrates $[26,27]$ and to study the coffeering effect in drops of polymer-nanocrystal mixtures [28]. Xie et al. [29] introduced an evaporation scheme to the multiphase LBM model and studied the effect of gravity on the shape of evaporating drops for different evaporation conditions and 
wetting properties of the substrate. Our present study is closely related to the work of Karapetsas et al. [30] who studied HTWs in evaporating sessile drops by implementing a "one-sided" two-dimensional (2D) numerical (FEM) model and using linear stability analysis. Their model demonstrated qualitative, but not quantitative, agreement with experimental observations.

The ultimate aim of current research is to achieve both qualitative and quantitative agreement between computer simulations and experiments. Achieving the first one would require a $3 \mathrm{D}$ numerical modeling, as the flow observed in drops $[19,21]$ is essentially three dimensional. Meanwhile 2D axisymmetric modeling is quite sufficient for the quantitative validation, because the most appropriate for that purpose and experimentally measurable quantity is the droplet's evaporation rate, which is weakly influenced by the $3 \mathrm{D}$ flow pattern inside a droplet.

The present paper is dedicated to the quantitative validation and, therefore, is limited to 2D axisymmetric numerical models, which take into account all relevant processes of heat and mass transfer, essential for the achievement of quantitative agreement with experiments. The original contribution comprises obtaining a semiempirical formula for the droplet's evaporation rate, increasing the accuracy of experimental video-data processing (improved fitting of a sessile droplet profile and correction due to noncircular contact perimeter) and, finally, computer simulations reproducing the conditions of three particular microgravity experiments. From theoretical and numerical points of view, current research is a substantial improvement of our previous work [31].

\section{THEORY}

In this section we derive a semiempirical theoretical formula predicting the rate of unsteady diffusion-limited evaporation for a nonisothermal pinned sessile droplet, taking into account Stefan flow in gas. A more rigorous theoretical consideration of nonisothermal evaporation of a spherical liquid droplet is given by Sobac et al. [32]. At the end of this section we present an expression, which well approximates the nonisothermal local vapor flux at the liquid-gas interface.

In what follows, we adopt the following assumptions: $p_{v \text {, sat }} \ll p_{\text {atm }}$, where $p_{v \text {, sat }}$ and $p_{\text {atm }}$ are saturated vapor and ambient atmospheric pressures, respectively; vapor-air mixture is considered as an ideal gas with constant density:

$$
\rho_{g}=\rho_{v}+\rho_{a}=\text { const, }
$$

where subscripts $g, v$, and $a$ stand for gas, vapor, and air, respectively.

\section{A. Steady-state isothermal diffusion}

Let us consider a spherically symmetric droplet of radius $L$ evaporating into ambient air. An isothermal $\left(T=\right.$ const $\left.=T_{\infty}\right) \quad$ steady-state $\quad$ (evaporated liquid is artificially replenished inside the droplet to keep its volume constant) model of diffusion-limited evaporation gives the following evaporative mass flux $J$ :

$$
J_{\mathrm{sph}}^{\mathrm{dif}}\left(L, T_{\infty}\right)=4 \pi D L\left[\rho_{v, \text { sat }}\left(T_{\infty}\right)-\rho_{v, \infty}\right],
$$

where dif and sph stand for "diffusive" and "spherical," respectively; $D=D\left(T_{\infty}\right)=$ const is a vapor-in-air diffusion coefficient; and $\rho_{v \text {, sat }}$ and $\rho_{v, \infty}$ are saturated and ambient vapor densities, respectively.

Below we consider several incremental modifications of Eq. (2) arising from increased problem complexity.

\section{B. Steady-state nonisothermal diffusion}

Moving from isothermal to nonisothermal diffusion requires accounting for the temperature dependence of vapor density $\rho_{v}(T)$ and of diffusion coefficient

$$
D(T)=A T^{3 / 2}
$$

where $A=\left(D p_{g}\right)_{\text {ref }} /\left(p_{g} T_{\text {ref }}^{3 / 2}\right)$ [33], gas pressure $p_{g}=p_{\text {atm }}$, and $\left(D p_{g}\right)_{\text {ref }}=1.337 \mathrm{Pam}^{2} / \mathrm{s}$ at $T_{\text {ref }}=298 \mathrm{~K}$ for ethanol vapor in air [34]. Even though the gas density $\rho_{g}$ in this problem is a function of temperature, we still can assume its constancy provided that

$$
\Delta \rho_{g} / \rho_{g}<\Delta \rho_{g} / \rho_{g, \min } \ll 1 \Rightarrow\left|T_{L}-T_{\infty}\right| / T_{\min } \ll 1,
$$

where $\Delta \rho_{g}=\rho_{g, \max }-\rho_{g, \text { min }}$. This problem can be easily solved analytically if convective heat and mass transfer is neglected in the gas phase. Thus, assuming constant thermal conductivity of gas, $k_{g}=$ const, and solving the diffusion and heat conduction equations with boundary conditions $\left.T\right|_{r=L}=T_{L}$ (here $r$ is a radial coordinate in a spherical system of coordinates), $\left.T\right|_{r=\infty}=T_{\infty},\left.\rho_{v}\right|_{r=L}=\rho_{v \text {, sat }}\left(T_{L}\right)$, and $\left.\rho_{v}\right|_{r=\infty}=\rho_{v, \infty}$, we get

$$
J_{\mathrm{sph}}^{\mathrm{dif}}\left(L, T_{L}\right)=4 \pi D_{\mathrm{eff}} L\left[\rho_{v, \text { sat }}\left(T_{L}\right)-\rho_{v, \infty}\right],
$$

where effective diffusion coefficient $D_{\text {eff }}=D\left(T_{\text {eff }}\right)=$ $A T_{\text {eff }}^{3 / 2}$ and effective temperature $T_{\text {eff }}=\left[\sqrt{T_{L} T_{\infty}}\left(\sqrt{T_{L}}+\right.\right.$ $\left.\left.\sqrt{T_{\infty}}\right) / 2\right]^{2 / 3}$. Note that in the limit $T_{L} \rightarrow T_{\infty}$ Eq. (5) turns into Eq. (2).

\section{Steady-state isothermal diffusion with Stefan flow}

Now let us extend the isothermal $\left(T=\right.$ const $\left.=T_{\infty}\right)$ diffusive model [see Eq. (2)] by considering an additional transport mechanism: steady-state spherically symmetric Stefan flow in the gas phase, which appears due to vapor production at the droplet surface (phase transition with a considerable change of substance density).

The gas flow is considered as an incompressible one, therefore the velocity vector is $\boldsymbol{u}_{g}^{\text {Stefan }}=\boldsymbol{r} u_{g}^{\text {Stefan }}(r) / r$, where $\boldsymbol{r}$ is the radius vector, $u_{g}^{\text {Stefan }}(r)=u_{0} L^{2} / r^{2}$, and $u_{0}=$ $u_{g}^{\text {Stefan }}(L)$. The following boundary conditions are applied here: $\left.\rho_{v}\right|_{r=L}=\rho_{v, \text { sat }}\left(T_{\infty}\right) ;\left.\rho_{v}\right|_{r=\infty}=\rho_{v, \infty}$; local density of vapor mass flux across the liquid-gas interface $j^{\text {Stefan }}=\left[\rho_{v} u_{0}-\right.$ $\left.D\left(\nabla \rho_{v}\right) \cdot \boldsymbol{n}\right]\left.\right|_{r=L}$, where $\boldsymbol{n}$ is the unit vector, normal to the interface and pointing into the gas phase and zero air flux across the interface $\left.\left[\rho_{a} u_{0}-D\left(\nabla \rho_{a}\right) \cdot \boldsymbol{n}\right]\right|_{r=L}=0$.

Combining these boundary conditions with Eq. (1), one can easily solve the vapor transport equation,

$$
\nabla \cdot\left(\rho_{v} \boldsymbol{u}_{g}^{\text {Stefan }}-D \nabla \rho_{v}\right)=0
$$


and get the following spherical droplet's evaporation rate:

$$
J_{\text {sph }}^{\text {Stefan }}\left(L, T_{\infty}\right)=4 \pi D L \rho_{g, L} \ln \left(\frac{\rho_{g, L}-\rho_{v, \infty}}{\rho_{g, L}-\rho_{v, \text { sat }}\left(T_{\infty}\right)}\right),
$$

where we used an additional subscript $L$ with the gas density $\rho_{g}$ to stress that $\rho_{g, L}=\left.\left(\rho_{v}+\rho_{a}\right)\right|_{r=L}$. This helps us to find the exact value of gas density in nonisothermal extensions of the current isothermal model. In what follows, $\rho_{g, L}$ will be calculated in accordance with the ideal gas law: $\rho_{g, L}=$ $p_{\text {atm }} M_{a} /\left(R T_{L}\right)$, where $M_{a}$ and $R$ are the molar mass of air and the universal gas constant, respectively. Equation (7) is a particular case of the Spalding model of combustion or evaporation of fuel droplets [35], which accounts for both diffusive and convective mass transport.

Note that the Stefan flow can be neglected $\left(u_{0} \rightarrow 0\right)$ for poorly volatile liquids, for which $\rho_{v} / \rho_{g} \ll 1$. In this limit, Eq. (7) turns into Eq. (2).

\section{Unsteady isothermal diffusion}

Solving an unsteady isothermal diffusion equation,

$$
\frac{\partial \rho_{v}}{\partial t}=D \frac{1}{r^{2}} \frac{\partial}{\partial r}\left(r^{2} \frac{\partial \rho_{v}}{\partial r}\right)
$$

with the boundary and initial conditions $L=$ const, $\left.\rho_{v}\right|_{r=L}=$ $\rho_{v, \text { sat }}\left(T_{\infty}\right),\left.\rho_{v}\right|_{r=\infty}=\rho_{v, \infty}$, and $\left.\rho_{v}\right|_{\substack{r>L \\ t=0}}=\rho_{v, \infty}$, we obtain the following droplet's evaporation rate:

$$
J_{\mathrm{sph}}^{\mathrm{dif}}\left(L, T_{\infty}, t\right)=4 \pi D L\left[\rho_{v, \text { sat }}\left(T_{\infty}\right)-\rho_{v, \infty}\right]\left(1+\frac{L}{\sqrt{\pi D t}}\right) .
$$

At $t \rightarrow \infty$ Eq. (9) becomes identical to Eq. (2). For more details, please see Appendix C.

\section{E. Unsteady evaporation of pinned sessile droplets}

Finally we empirically combine all the modifications [see Eqs. (5), (7), and (9)] of Eq. (2) in one formula. In order to move from a spherical to a sessile droplet, we first took a half of the spherical droplet's evaporation rate, which corresponds to a sessile droplet with contact angle $\theta=\pi / 2: J_{\pi / 2}=J_{\text {sph }} / 2$. Then, to take into account the dependence on contact angle $\theta$, we have replaced the geometrical parameter $L$ by the product $L F(\theta)$, where $F(\theta)$ is a well known function of contact angle, derived by Picknett and Bexon [36] (see also Ref. [37]), and equivalent to another one, $F_{P}(\theta)=2 F(\theta)$, derived later by Popov [38]:

$$
F_{P}(\theta)=\frac{\sin \theta}{1+\cos \theta}+4 \int_{0}^{\infty} \frac{1+\cosh (2 \theta \tau)}{\sinh (2 \pi \tau)} \tanh [(\pi-\theta) \tau] d \tau .
$$

Note that $F(0)=2 / \pi$, and $F(\pi / 2)=1$. Instead of temperature $T_{L}$ we have used the average temperature of sessile droplet's surface $\Gamma: T_{\mathrm{av}}=\left(\int_{\Gamma} T d \Gamma\right) /\left(\int_{\Gamma} d \Gamma\right)$, which is proven to be a proper modeling approach $[37,39]$.

Thus, our final semiempirical expression of an unsteady diffusion-limited evaporation rate for a nonisothermal pinned sessile droplet, accounting for Stefan flow in gas, takes the form

$$
\begin{aligned}
& J^{\text {Stefan }}\left(L, T_{\mathrm{av}}, \theta(t), t\right) \\
& =2 \pi D_{\mathrm{eff}}^{*} L F(\theta(t)) \\
& \quad \times\left(1+\frac{L F(\theta(t))}{\sqrt{\pi D_{\mathrm{eff}}^{*} t}}\right) \rho_{g}^{*} \ln \left(\frac{\rho_{g}^{*}-\rho_{v, \infty}}{\rho_{g}^{*}-\rho_{v, \text { sat }}^{*}}\right),
\end{aligned}
$$

where $L$ now is a constant contact line radius, whereas $\theta$ changes with time; the asterisk stands for a dependence on $T_{\mathrm{av}}: D_{\mathrm{eff}}^{*}=D\left(T_{\mathrm{eff}}^{*}\right)$ [see Eq. (3)]; $T_{\text {eff }}^{*}=\left[\sqrt{T_{\mathrm{av}} T_{\infty}}\left(\sqrt{T_{\mathrm{av}}}+\right.\right.$ $\left.\left.\sqrt{T_{\infty}}\right) / 2\right]^{2 / 3} ; \rho_{v \text {,sat }}^{*}=\rho_{v \text {,sat }}\left(T_{\mathrm{av}}\right) ; \rho_{g}^{*}=p_{\mathrm{atm}} M_{a} /\left(R T_{\mathrm{av}}\right)$. Equation (11) agrees well with the results of an equivalent numerical model, as will be shown below.

For a purely diffusive model, which does not take into account the Stefan flow:

$$
\begin{aligned}
J^{\mathrm{dif}}\left(L, T_{\mathrm{av}}, \theta(t), t\right)= & 2 \pi D_{\mathrm{eff}}^{*} L F(\theta(t))\left(1+\frac{L F(\theta(t))}{\sqrt{\pi D_{\mathrm{eff}}^{*}}}\right) \\
& \times\left(\rho_{v, \text { sat }}^{*}-\rho_{v, \infty}\right) .
\end{aligned}
$$

Usually the temperature $T_{\mathrm{av}}$ is not available theoretically or experimentally, therefore in Eqs. (11) and (12) we have used $T_{\text {av }}$ from our computer simulations (note that numerically obtained $T_{\mathrm{av}}$ is not a fitting parameter, since computer simulations do not include any fitting parameters). However, for relatively thin droplets $T_{\mathrm{av}}$ can be estimated from the following simplified energy balance equation: $k_{l} \frac{T_{s}-T_{\mathrm{av}}}{h_{\mathrm{av}}} S_{d}=$ $P_{\mathrm{ev}}$, where $k_{l}$ is the droplet's thermal conductivity, $T_{s}$ is the temperature of substrate-droplet interface (equal to the substrate's temperature for highly heat conductive substrates), $S_{d}=\pi L^{2}$ is the droplet's contact area, $P_{\mathrm{ev}}$ is the evaporative power (rate of heat consumption by evaporation), and $h_{\mathrm{av}}$ is the average droplet's height which can be estimated as $h_{\mathrm{av}}=V_{d} / S_{d}$, where $V_{d}$ is the droplet's volume.

Regarding the time-dependent contact angle $\theta(t)$, if it is not possible to predict it theoretically, then one should use its experimentally available value. Nevertheless, if $\theta$ remains below $40^{\circ}(\theta<2 \pi / 9)$, then the function $F(\theta)$ can be assumed a constant $\left.F(\theta)\right|_{\theta<2 \pi / 9} \approx 2 / \pi$.

\section{F. Approximation of the surface density of evaporative mass flux}

In this subsection we present a formula, which well approximates the local surface density, $j$, of nonisothermal evaporative mass flux across the liquid-gas interface. This formula can be used as a boundary condition in one-sided models of evaporating sessile droplets, especially in 3D computer simulations, when employing the two-sided model is computationally expensive. Only the temperature and tangential velocity at the droplet surface, which are readily available in one-sided models, will be used as input data for this approximation. The values of two fitting parameters, which are required for our approximation, will be obtained through fitting an evaporation flux $j$ available numerically from our two-sided 2D model.

In what follows we assume that liquid-gas interface is a spherical cap with contact angle $\theta \leqslant \pi / 2$ (see Fig. 3), $\Gamma: r=$ $f(z)=\sqrt{R_{d}^{2}-\left(z-z_{d}\right)^{2}}$ for $0 \leqslant z \leqslant R_{d}+z_{d}$, where $r$ and $z$ 
are correspondingly radial and vertical cylindrical coordinates ( $z=0$ at the droplet-substrate interface), $R_{d}=L / \sin \theta$, and $z_{d}=-R_{d} \cos \theta$.

In such geometry, the exact solution $j(r, \theta)$ was reported earlier for the case of isothermal steady-state diffusion-limited evaporation with no Stefan flow; see for example Eq. (A2) in [40] or Eq. (A5) in [38]. This solution was given in an integral form with a Legendre function of the first kind in the integrand, which is quite difficult to compute and analyze. Deegan et al. [40] suggested a convenient approximation to the exact solution:

$$
j_{\text {Deegan }}(r, \theta)=j_{0}\left[1-(r / L)^{2}\right]^{-\lambda} \text { for } 0 \leqslant \theta \leqslant \pi / 2,
$$

where $\lambda=(\pi-2 \theta) /(2 \pi-2 \theta)$ and $j_{0}=j(0, \theta)$. Equation (13) becomes an exact solution, which implies zero approximation error, in two particular cases: $\theta=0$ (see Appendix A for more information) and $\theta=\pi / 2$ [uniform flux distribution $j(r, \pi / 2)=j_{0}$ due to spherical symmetry of the problem].

According to authors [40], Eq. (13) gives maximum approximation error of $10 \%$. However, our numerical estimates of the exact solution [Eq. (A5) in [38]] with a computation error less than $0.1 \%$ show that Eq. (13) in fact gives an approximation error above $10 \%$ for a wide range of $r$ and $\theta$ values. In particular, for contact angle $\theta=\pi / 3$ at $r / L>0.99$, Deegan's approximation gives an error above $34 \%$. Due to this, using our numerical estimates of the exact solution, we have constructed a more accurate approximation, $j_{i}(r, \theta)$ (the subscript $i$ stands for isothermal), with an approximation error less than $1 \%$. This new approximation is presented below:

$$
j_{i}(r, \theta)=j_{0} \chi^{-\lambda}(1-\omega) \text { for } 0 \leqslant \theta \leqslant \pi / 2,0 \leqslant r<L,
$$

where

$$
\begin{aligned}
\chi= & 1-(r / L)^{2}, \\
j_{0}= & j(0, \theta)=\frac{D}{L}\left(\rho_{v, \text { sat }}-\rho_{v, \infty}\right) G(\theta)=\frac{J G(\theta)}{2 \pi L^{2} F(\theta)}, \\
G(\theta)= & 0.008348 \theta^{4}-0.1026 \theta^{3}+0.001815 \theta^{2}+0.4491 \theta+0.6368, \\
F(\theta)= & \left\{\begin{array}{ll}
\left(0.6366 \theta+0.09591 \theta^{2}-0.06144 \theta^{3}\right) / \sin \theta, \quad 0 \leqslant \theta<\pi / 18 \\
\left(0.00008957+0.6333 \theta+0.116 \theta^{2}\right. \\
\left.-0.08878 \theta^{3}+0.01033 \theta^{4}\right) / \sin \theta, \quad \pi / 18 \leqslant \theta \leqslant \pi
\end{array}, \quad \text { (hyperbola) },\right. \\
\omega= & \begin{cases}H\left[3-\sqrt{\left.4+5\left(1-\theta / \theta_{H E}\right)^{2}\right],}\right. \\
-E+\sqrt{\lambda_{E}^{2} E^{2}+\left(1-\lambda_{E}^{2}\right)(H+E)^{2},} \quad \theta_{H E}<\theta \leqslant \pi / 2 \quad \text { (ellipse) }\end{cases} \\
\lambda_{E}= & \left(2 \theta-2 \theta_{H E}\right) /\left(\pi-2 \theta_{H E}\right), \\
\theta_{H E}= & 0.7864 H+0.9103, \\
E= & -2.679 H+0.7265, \\
H= & 0.26\left(1-\chi^{0.7}\right) .
\end{aligned}
$$

Like the Deegan's approximation, $j_{i}(r, \theta)$ also becomes an exact solution in two particular cases: $\theta=0$ and $\theta=\pi / 2$. If one takes $\omega=0$, then approximation (14) becomes identical to Deegan's approximation (13).

Let us use the isothermal flux distribution (14) as a base for obtaining the approximation of the nonisothermal one. First of all, we will substitute the most general form of total evaporation rate $J^{\operatorname{Stefan}}\left(L, T_{\mathrm{av}}, \theta(t), t\right)$ into Eq. (15) instead of $J$, which ensures that local vapor flux, being integrated over the droplet surface, matches the total vapor flux:

$$
\begin{aligned}
j_{0}^{*} & =\frac{J^{\operatorname{Stefan}}\left(L, T_{\mathrm{av}}, \theta(t), t\right) G(\theta)}{2 \pi L^{2} F(\theta)}, \\
j_{i}^{*}(r, \theta) & =j_{0}^{*} \chi^{-\lambda}(1-\omega) .
\end{aligned}
$$

This flux distribution can be considered as zero approximation of the nonisothermal one, as if temperature was uniform along the droplet surface and equal $T_{\mathrm{av}}$. Now we introduce a small perturbation into this flux due to a small deviation of local temperature from $T_{\mathrm{av}}$ :

$$
j(r, \theta, T)=j_{i}^{*}(r, \theta)\left[1+B\left(T-T_{\mathrm{av}}\right)\right],
$$

where $B$ is the first fitting parameter in our nonisothermal approximation. In the original unperturbed isothermal problem there exists vapor flux only in the normal direction with respect to the droplet surface, and there is no flux in the tangential direction due to the uniform temperature and correspondingly uniform vapor density along the droplet surface. Therefore $j_{i}^{*}(r, \theta)$ represents only a normal component of the vapor flux, which means that introduced perturbation gives a correction which represents the contribution of only the normal component of the flux. However in the nonisothermal problem tangential fluxes also appear due to tangential liquid flow and nonzero gradient of vapor density along the droplet surface. These tangential fluxes, emerging in the nonisothermal problem, should also contribute to the local evaporation rate.

In order to estimate this additional contribution $j_{+}$to the local evaporation rate, let us introduce a thin boundary layer of thickness $\Delta h$ in the gas phase above the droplet surface, in which tangential vapor fluxes $\boldsymbol{j}_{\tau}=-D \nabla_{\Gamma} \rho_{v}+\rho_{v} \boldsymbol{u}_{\Gamma}$ [where $\nabla_{\Gamma}=\nabla-(n \otimes n) \nabla$ and $\boldsymbol{u}_{\Gamma}=\boldsymbol{u}-(\boldsymbol{n} \otimes \boldsymbol{n}) \boldsymbol{u}$ is the tangent velocity vector at the liquid-gas interface, $\boldsymbol{n} \otimes \boldsymbol{n}$ is the rotation matrix to move from the global reference frame to the local 


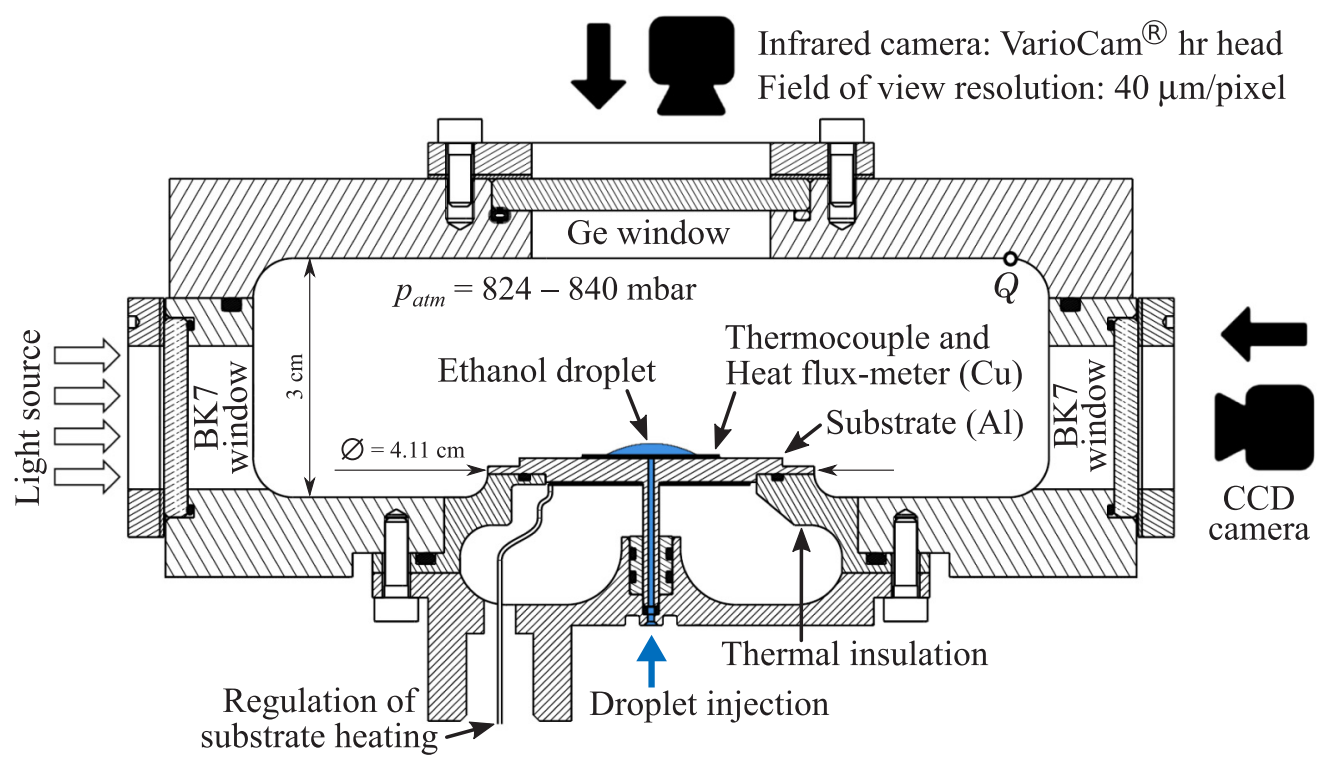

FIG. 1. Experimental setup based on previous work [19].

one] are balanced by the flux $j_{+}$across the bottom surface of the boundary layer (liquid-gas interface). Thus, considering a portion of boundary layer as a control volume with bottom area $S$ and height $\Delta h$, we can write an integral equation for this balance:

$$
\iint_{S} j_{+} d S=\Delta h \oint_{\partial S} \boldsymbol{j}_{\tau} \cdot[\boldsymbol{d} \boldsymbol{l} \times \boldsymbol{n}],
$$

where $\boldsymbol{d} \boldsymbol{l}$ is a directed line element of the closed curve $\partial S$, which bounds the area $S$. Vector $[\boldsymbol{d} \boldsymbol{l} \times \boldsymbol{n}]$ is perpendicular to the side surface of the control volume and pointing outwards. Applying the curl theorem to the right side of Eq. (18) we obtain

$$
\begin{aligned}
j_{+} & =\Delta h\left[\nabla \times\left[\boldsymbol{n} \times \boldsymbol{j}_{\tau}\right]\right] \cdot \boldsymbol{n}=\Delta h[\nabla-(\boldsymbol{n} \otimes \boldsymbol{n}) \cdot \boldsymbol{\nabla}] \cdot \boldsymbol{j}_{\tau} \\
& =\Delta h \nabla_{\Gamma} \cdot \boldsymbol{j}_{\tau} .
\end{aligned}
$$

The right hand side of Eq. (19) is the surficial divergence of the tangent vector $\boldsymbol{j}_{\tau}$, multiplied by the boundary layer thickness $\Delta h$, which will be the second fitting parameter in our approximation.

Finally, the resulting approximation of the local evaporation rate, including both contributions, takes the following form:

$$
\begin{aligned}
j_{\text {approx }}= & j(t, \theta, T)+j_{+}=j_{i}^{*}(r, \theta)\left[1+B\left(T-T_{\mathrm{av}}\right)\right] \\
& +\Delta h \nabla_{\Gamma} \cdot \boldsymbol{j}_{\tau} .
\end{aligned}
$$

In the case of the axisymmetric problem the last term of Eq. (20) simplifies, and in cylindrical coordinates $(r, z)$ we obtain

$$
j_{\text {approx }}=j_{i}^{*}(r, \theta)\left[1+B\left(T-T_{\mathrm{av}}\right)\right]+\Delta h \frac{n_{z}}{r} \frac{d\left(r j_{\tau}\right)}{d r},
$$

where $n_{z}$ is the vertical component of the normal vector $\boldsymbol{n}$, and $j_{\tau}=\left|\boldsymbol{j}_{\tau}\right|$ is considered as a function of coordinate $r$ only. Two fitting parameters $B$ and $\Delta h$ are obtained from our two-sided $2 \mathrm{D}$ numerical models and presented in the Results section.

\section{EXPERIMENTS}

Our theoretical and numerical models are validated against experimental data obtained under microgravity conditions during parabolic flight experiments on board of Novespace A300-ZeroG aircraft in Bordeaux, France.

Sessile drops of ethanol evaporated from a heated substrate into a closed chamber filled with air under measured cabin pressure; see Fig. 1. The contact line stayed pinned during all periods of evaporation in microgravity. The heat flux meter (15 $\mathrm{mm}$ diameter and $0.6 \mathrm{~mm}$ thickness, copper body) was covered with a thin $(13.9 \mathrm{~nm})$ layer of perfluorocarbon (PFC) to induce a good wetting and prevent droplet detachment during its injection through a tube of diameter $0.7 \mathrm{~mm}$ at the center of the substrate. In the present work we consider three particular experiments with substrate temperature $T_{s}$ at $5.4,15.1$, and $20.0 \mathrm{~K}$ above the measured wall temperature of the experimental cell (measurement and regulation precision is $\pm 0.1 \mathrm{~K}$ ).

The infrared (IR) camera (3-5- $\mu \mathrm{m}$ wavelength) recorded the droplet's top view to capture hydrothermal instabilities, whereas a charge-coupled device (CCD) camera captured its side-view geometry in the visible spectrum. The contact angle $\theta$, radius $L$, and droplet's volume $V$ are extracted from visual data by fitting the droplet profile with a spherical cap outline taking into account camera tilt. In addition, the correction of the contact radius $L$ has been introduced as discussed below.

The shape of a real sessile droplet is never axially symmetric due to the nonideality of real experimental systems (especially in nonstable parabolic flight conditions). Figure 2 shows one of the biggest experimentally observed deviations from the circular shape, which is chosen here for a demonstrative purpose. Nevertheless, this deviation is not so big to be directly modeled. Instead, it can be treated as a systematic experimental error for each particular experiment, because the shape is preserved during the whole evaporation period due to contact line pinning. This systematic error results in a difference between visible and real average contact radii $L$ (see Fig. 2), 


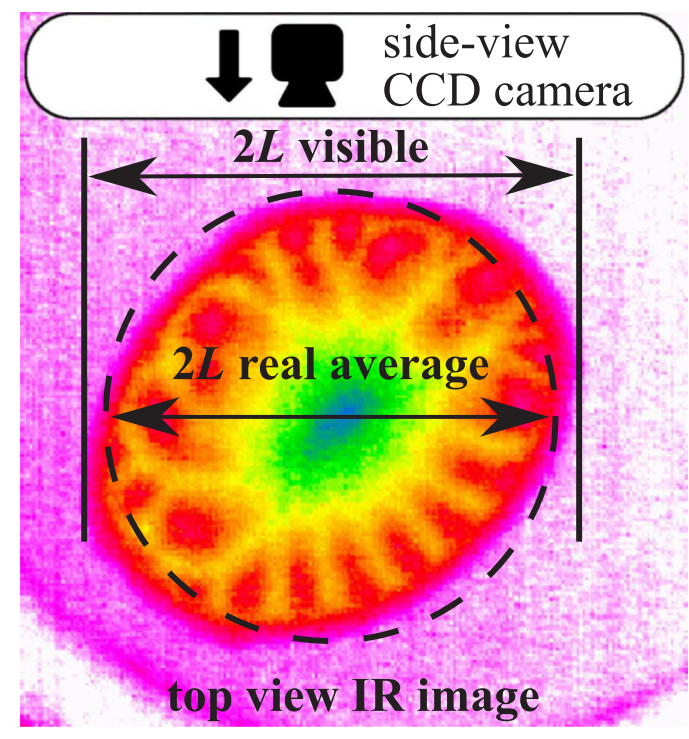

FIG. 2. Visible and real average contact radii. The dashed line shows a circle with the same area as the one of droplet's base.

and can be corrected by replacing visible contact radius $L$ with the real average one, which is obtained by fitting the real contact area (available from the top view image) with $\pi L^{2}$. This approach corrects the systematic error and, thus, increases the consistency between an axisymmetric model and the experimental data.

In addition to the visually available droplet's geometry, we have used a heat flux meter to extract the droplet's evaporation rate indirectly. Experimentally measured heat flux through the substrate was decomposed on radiative [radiated power $P_{\mathrm{rad}}=\epsilon_{f} \sigma_{S B}\left(T_{s}^{4}-T_{\infty}^{4}\right) S_{f}$, where $\sigma_{S B}$ is the Stefan-Boltzmann constant, $S_{f}$ and $\epsilon_{f}=0.95$ are flux-meter area and emissivity, respectively], and conductive (through the air, available numerically: $1.5,4.6$, and $6.0 \mathrm{~mW}$ for $T_{s}-$ $T_{\infty}=5.4,15.1$, and $20.0 \mathrm{~K}$, respectively) and evaporative components. The convective component is absent due to the absence of natural convection under microgravity conditions. Then experimental rate of evaporation was calculated as $J_{\mathrm{exp}}=P_{\mathrm{ev}} M_{e} / \Lambda$, where $P_{\mathrm{ev}}$ is the evaporative power, $\Lambda$ and $M_{e}$ are the latent heat of vaporization (in $\mathrm{J} / \mathrm{mol}$ ) and the molar mass of evaporating substance (ethanol), respectively.

More information on experimental techniques can be found in the work by Carle et al. [19].

\section{COMPUTER SIMULATIONS}

We have developed an axisymmetric numerical model of unsteady evaporation of pinned sessile drops of ethanol from a heated substrate into a closed chamber. The commercial software COMSOL MULTIPHYSICS has been used for this purpose. It uses the finite element method (with quadratic Lagrangian elements) to discretize governing equations in their weak formulation, while all boundary conditions are implemented in the form of constraints with Lagrange multipliers. We applied arbitrary Lagrangian-Eulerian (ALE) and interface tracking methods to model moving mesh which follows the motion of the liquid-gas interface.

\section{A. Problem geometry}

The origin of cylindrical coordinates $(r, z)$ is located in the plane of the substrate-droplet interface, and the vertical axis coincides with the axis of the droplet's symmetry. Though the real experimental cell has a square shape and not the axisymmetric one, its size is much bigger than the size of the evaporating droplet located in the cell center. This allows us to neglect the shape and take into account only the cell's volume. Thus, in our numerical model we have created an axisymmetric chamber of the same height $(3 \mathrm{~cm})$ as the experimental one, while its radius $(5.63 \mathrm{~cm})$ was chosen to match the experimental cell volume $\left(294 \mathrm{~cm}^{3}\right)$. The geometry of substrate and heat flux meter was identical to the experimental one (see Fig. 1).

\section{B. Material properties}

The properties of materials in the model were identical to the experimental ones [33]: aluminium for the substrate, copper for the heat flux-meter, and pure ethanol for the droplet. The ethanol surface tension is $\gamma=20.6 \mathrm{mN} / \mathrm{m}$, while its temperature coefficient is $d \gamma / d T=-0.082 \mathrm{mN} /(\mathrm{m} \mathrm{K})$. The latent heat of vaporization was taken as a function of temperature: $\Lambda(T)=c_{1}\left(1-T / T_{c}\right)^{c_{2}}$, where $c_{1}=55789$ $\mathrm{J} / \mathrm{mol}, c_{2}=0.31245$, and $T_{c}=514 \mathrm{~K}$. According to the Clausius-Clapeyron equation, the saturated vapor pressure of ethanol

$$
p_{\mathrm{sat}}(T)=p_{\mathrm{sat}}^{\mathrm{ref}} \exp \left[-\frac{\Lambda(T)}{R}\left(\frac{1}{T}-\frac{1}{T_{\mathrm{sat}}^{\mathrm{ref}}}\right)\right],
$$

where $p_{\text {sat }}^{\text {ref }}=13838 \mathrm{~Pa}, T_{\text {sat }}^{\text {ref }}=308.15 \mathrm{~K}$, and $R$ is the universal gas constant. The influence of the droplet's surface curvature on $p_{\text {sat }}(T)$ can be neglected [41]. Since the temperature change occurs mostly in the air domain (the medium with lowest heat conductivity in the system), its dynamic viscosity $\mu_{a}$, heat conductivity $k_{a}$, and vapor diffusivity $D$, are taken as functions of local temperature: $\mu_{a}(T)$ and $k_{a}(T)$ are determined according to Sutherland's law:

$$
\begin{gathered}
\mu_{a}(T)=\mu_{\text {ref }}\left(T / T_{\text {ref }}\right)^{3 / 2}\left(T_{\text {ref }}+T_{\mu}\right) /\left(T+T_{\mu}\right), \\
k_{a}(T)=k_{\text {ref }}\left(T / T_{\text {ref }}\right)^{3 / 2}\left(T_{\text {ref }}+T_{k}\right) /\left(T+T_{k}\right),
\end{gathered}
$$

where $\mu_{\text {ref }}=17.16 \mu \mathrm{Pas} ; k_{\text {ref }}=24.14 \mathrm{~mW} /(\mathrm{m} \mathrm{K}) ; T_{\text {ref }}, T_{\mu}$, and $T_{k}$ are respectively $273.15,110.4$, and $194.4 \mathrm{~K}$. For $D(T)$ see Eq. (3).

The adsorption of water from air on the ethanol surface is neglected, because air humidity becomes relatively low due to droplet heating.

\section{Bulk equations}

In accordance with ALE formulation, the following governing equations describe transport phenomena in the bulk. The heat transfer is

$$
\rho c_{p}\left(\left.\frac{\partial T}{\partial t}\right|_{m}+(\boldsymbol{u}-\boldsymbol{w}) \cdot \nabla T\right)=\nabla \cdot(k \nabla T),
$$

where the subscript $m$ means that the time derivative is taken at a fixed mesh node (fixed mesh coordinates), $\boldsymbol{w}$ is the mesh velocity, $\nabla$ is the nabla operator, $\rho, c_{p}$, and $k$ are respectively 
density, specific heat capacity at constant pressure, and thermal conductivity of the corresponding phase. The bulk velocity $\boldsymbol{u}=0$ for solids.

Continuity and incompressible Navier-Stokes equations under the Boussinesq approximation in liquid and gas are

$$
\begin{gathered}
\nabla \cdot \boldsymbol{u}=0 \\
\rho\left(\left.\frac{\partial \boldsymbol{u}}{\partial t}\right|_{m}+[(\boldsymbol{u}-\boldsymbol{w}) \cdot \nabla] \boldsymbol{u}\right)=\nabla \cdot \boldsymbol{\sigma}+\boldsymbol{F}_{B},
\end{gathered}
$$

where $\sigma=-p \boldsymbol{I}+\boldsymbol{\pi}$ is the total stress tensor, $p$ is the hydrodynamic pressure, $\boldsymbol{I}$ is the identity tensor, and $\boldsymbol{\pi}=$ $\mu\left[\nabla \boldsymbol{u}+(\nabla \boldsymbol{u})^{\mathrm{T}}\right]$ is the viscous stress tensor. The buoyancy force $\boldsymbol{F}_{B}=\boldsymbol{g} \Delta \rho$ is zero under microgravity conditions ( $\boldsymbol{g}=$ $0)$, while for nonzero gravity $(g \neq 0)$ it accounts for the density change $\Delta \rho$ due to liquid-gas thermal expansion as well as due to the vapor content in the gas. In the case of modeling pure diffusion (without Stefan or natural convection in gas) Eqs. (26) and (27) are not solved in the gas.

Under assumption (1) the equation of vapor diffusion convection in the gas phase reads

$$
\left.\frac{\partial \rho_{v}}{\partial t}\right|_{m}+\left(\boldsymbol{u}_{g}-\boldsymbol{w}\right) \cdot \nabla \rho_{v}=\nabla \cdot\left(D \nabla \rho_{v}\right),
$$

where $\boldsymbol{u}_{g}=0$ if Stefan or natural convection is absent.

\section{Initial and boundary conditions}

The initial temperature is $\left.T\right|_{t=0}=T_{\infty}$ in the gas phase and $\left.T\right|_{t=0}=T_{s}$ in the substrate, heat flux-meter, and droplet. The initial velocity is $\left.\boldsymbol{u}\right|_{t=0}=0$ in gas and liquid. The initial pressure is $\left.p_{g}\right|_{t=0}=p_{\text {atm }}$ in gas and $\left.p_{l}\right|_{t=0}=p_{\text {atm }}+2 \gamma \sin \theta_{0} / L$ in liquid, where $\theta_{0}$ is the initial contact angle. The initial vapor density in gas is $\left.\rho_{v}\right|_{t=0}=0$.

Boundary conditions of axial symmetry are applied at $r=0$ for all fields. The temperature $T_{\infty}$ is imposed at the chamber wall, and $T_{s}$ is at the interface between the heat flux meter and aluminium substrate. Zero normal heat flux $(\boldsymbol{n} \cdot \boldsymbol{\nabla}=0)$ is on the surface of thermally insulating material (see Fig. 1). The conditions of temperature and heat flux continuity are applied at liquid-solid and gas-solid interfaces. The vapor is saturated at the liquid-gas (lg) interface (see Appendix B for a justification of this boundary condition): $\left(\rho_{v}\right)_{\mathrm{lg}}=\rho_{v \text {, sat }}(T)=M_{e} p_{\text {sat }}(T) /(R T)$, where $M_{e}$ is the molar mass of evaporating substance (ethanol), and $p_{\text {sat }}(T)$ is given by Eq. (22). No penetration of vapor is applied at all gas-solid (gs) interfaces: $\left(\boldsymbol{n} \cdot \nabla \rho_{v}\right)_{g s}=0$.

The no-slip and no-penetration boundary conditions are applied for Eqs. (26) and (27) at the liquid-solid (1s) and gassolid interfaces: $\boldsymbol{u}_{\mathrm{ls}}=0, \boldsymbol{u}_{g s}=0$. Constant pressure is set in a specific point $Q$ (see Fig. 1) of the gas phase $\left(p_{g}\right)_{Q}=$ const $=$ $p_{\text {atm. }}$.

Now let us consider the interfacial conditions for the moving liquid-gas interface $\Gamma$ (see Fig. 3) with normal unit vector $\boldsymbol{n}$ pointing into the gas phase and tangent unit vector $\boldsymbol{\tau}$. Let $j$ be the local surface density of evaporative mass flux [in $\left.\mathrm{kg} /\left(\mathrm{m}^{2} \mathrm{~s}\right)\right]$ across the interface $\Gamma$ in the direction of vector $\boldsymbol{n}$, and $u_{\Gamma}$ be the normal velocity of the interface itself in the direction of vector $\boldsymbol{n}$.

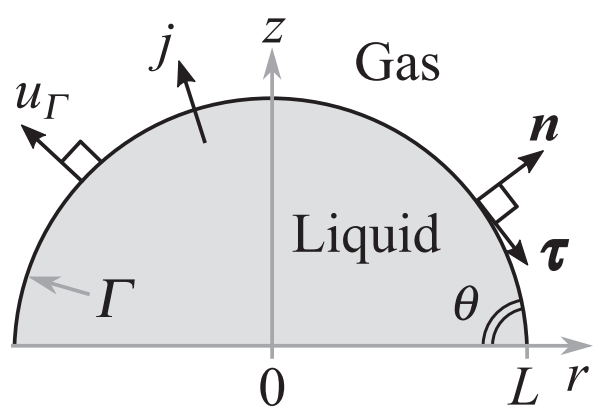

FIG. 3. Liquid-gas interface.

At the interface $\Gamma$ we assumed temperature continuity $\left(T_{l}=\right.$ $T_{g}$ ) and heat flux discontinuity:

$$
k_{g}(\nabla T)_{g} \cdot \boldsymbol{n}-k_{l}(\nabla T)_{l} \cdot \boldsymbol{n}=j \Lambda(T) / M_{e} .
$$

The boundary condition for the normal liquid velocity at $\Gamma$ reads (expressions for $j$ and $u_{\Gamma}$ will be given later)

$$
\rho_{l}\left(\boldsymbol{u}_{l} \cdot \boldsymbol{n}-u_{\Gamma}\right)=j
$$

The stress balance at $\Gamma$ is used to obtain boundary conditions for pressure and tangent velocity in the liquid phase:

$$
(\boldsymbol{\sigma} \cdot \boldsymbol{n})_{l}-(\boldsymbol{\sigma} \cdot \boldsymbol{n})_{g}=-\gamma(\nabla \cdot \boldsymbol{n})_{\Gamma} \boldsymbol{n}+\frac{d \gamma}{d T}\left(\nabla_{\Gamma} T\right),
$$

where $\nabla_{\Gamma} T=(\nabla-\boldsymbol{n} \boldsymbol{n} \cdot \nabla) T$ is the surface gradient of the temperature and $(\boldsymbol{\nabla} \cdot \boldsymbol{n})_{\Gamma}$ is the divergence of the vector $\boldsymbol{n}$ at $\Gamma$, that is the curvature of $\Gamma$, which is equal to $2 \sin \theta / L$. Taking the scalar product of Eq. (31) with vector $\tau$ and neglecting the viscous stress in the gas phase $\left(\mu_{g} / \mu_{l} \ll 1\right)$ we obtain the boundary condition for the thermal Marangoni convection which determines the tangent liquid velocity. A similar procedure with vector $\boldsymbol{n}$ results in the boundary condition for liquid pressure at $\Gamma$.

In the case of only diffusive vapor transport (when the gas flow is neglected), under assumption (1) $j$ is determined as $j=\left(-D \nabla \rho_{v}\right) \cdot \boldsymbol{n}$. Let us derive $j$ in the case when convective vapor transport is also present. In this case

$$
j=\rho_{v}\left(\boldsymbol{u}_{g} \cdot \boldsymbol{n}-u_{\Gamma}\right)-D \nabla \rho_{v} \cdot \boldsymbol{n},
$$

while air transport across the interface $\Gamma$ is zero:

$$
0=\rho_{a}\left(\boldsymbol{u}_{g} \cdot \boldsymbol{n}-u_{\Gamma}\right)-D \nabla \rho_{a} \cdot \boldsymbol{n} .
$$

Excluding the term $\left(\boldsymbol{u}_{g} \cdot \boldsymbol{n}-u_{\Gamma}\right)$ from Eqs. (32) and (33) and using Eq. (1) we obtain $j$ for the case of diffusive-convective vapor transport:

$$
j=\frac{\left(-D \nabla \rho_{v}\right) \cdot \boldsymbol{n}}{1-\rho_{v} / \rho_{g}} .
$$

Summing Eqs. (32) and (33) and using Eq. (1), we derive the boundary condition for the normal gas velocity at the interface $\Gamma$, which is similar to Eq. (30):

$$
\rho_{g}\left(\boldsymbol{u}_{g} \cdot \boldsymbol{n}-u_{\Gamma}\right)=j .
$$

The tangent gas velocity at $\Gamma$ is determined by the assumed no-slip boundary condition:

$$
\boldsymbol{u}_{g} \cdot \boldsymbol{\tau}=\boldsymbol{u}_{l} \cdot \boldsymbol{\tau}
$$


Finally, the expression for $u_{\Gamma}$ can be derived based on the total evaporative mass flux $J=\int_{\Gamma} j d \Gamma$ and two assumptions: the pinned contact line ( $L=$ const) and the spherical-cap shape of the droplet in the course of evaporation $[\Gamma: r=$ $f(z)=\sqrt{R_{d}^{2}-\left(z-z_{d}\right)^{2}}$ for $0 \leqslant z \leqslant R_{d}+z_{d}$, where $R_{d}=$ $L / \sin \theta$ and $\left.z_{d}=-R_{d} \cos \theta\right]$ :

$$
u_{\Gamma}=-\frac{J}{\pi \rho_{l} L^{3}} \frac{(1+\cos \theta)^{2}}{\sin \theta} z .
$$

The current model does not employ any physical mechanisms to overcome the problem of mathematical singularity of $j$ at the contact line, because this singularity is integrable ( $J$ is mathematically finite) and the size of singularity region (see Appendix B for the estimation of this size), where different physics comes into play, is a few orders of magnitude less than the size of the finite elements used. It means that this singularity region is out of numerical resolution (underresolved) and gives an insignificant and negligible contribution to the overall numerical solution. Taking into account real contact line physics would require computationally expensive and unnecessary mesh refinement by a few orders of magnitude near the contact line. In terms of discrete quantities, singularity never shows up numerically, because the discretized value of $\nabla \rho_{v}$ is never infinite due to the nonzero distance between discrete nodes of $\rho_{v}$. In this way singularity is smoothed (underresolved) numerically. Viscous stress singularity is also smoothed (underresolved) numerically and was not treated in any special way. The mesh is refined around the contact line (the element size is $10^{-2} L$ ) to reduce the area of underresolved solution. The growth rate for mesh elements is less than 1.1 in the whole computational domain.

\section{E. Computational procedure}

The air pressure inside of the chamber, temperatures $T_{s}$ (substrate) and $T_{\infty}$ (chamber wall), as well as the initial droplet geometry $\left(L_{0}\right.$ and $\left.\theta_{0}\right)$ were chosen as in three particular experiments: (1) $p_{\mathrm{atm}}=840 \mathrm{mbar}, T_{s}=305.8 \mathrm{~K}, T_{\infty}=300.4$ $\mathrm{K}, L_{0}=3.71 \mathrm{~mm}, \theta_{0}=19.3^{\circ}$; (2) $p_{\mathrm{atm}}=824 \mathrm{mbar}, T_{s}=$ $315.8 \mathrm{~K}, T_{\infty}=300.7 \mathrm{~K}, L_{0}=2.90 \mathrm{~mm}, \theta_{0}=17.3^{\circ}$; (3) $p_{\text {atm }}=837$ mbar, $T_{s}=320.8 \mathrm{~K}, T_{\infty}=300.8 \mathrm{~K}, L_{0}=3.15$ $\mathrm{mm}, \theta_{0}=18.2^{\circ}$.

The computational process is started with a preheating stage $(20 \mathrm{sec})$, as in experiments, to set the temperature field in the chamber. During this stage the droplet evaporation, as well as liquid and gas flow, are switched off numerically.

Next, if the gravity is nonzero in our computer simulation, then during next $20 \mathrm{sec}$ the gas flow is switched on (along with the heat transport) in order to establish a stationary natural convection and corresponding temperature field in the chamber.

After that, all physical processes are switched on, and evaporation starts instantaneously, accompanied by developing thermocapillary flow in the droplet. This last computational stage is validated against theory and experiments.

\section{RESULTS AND DISCUSSION}

Using the experimentally recorded evolution of a droplet's geometry was insufficient for the extraction of its evaporation

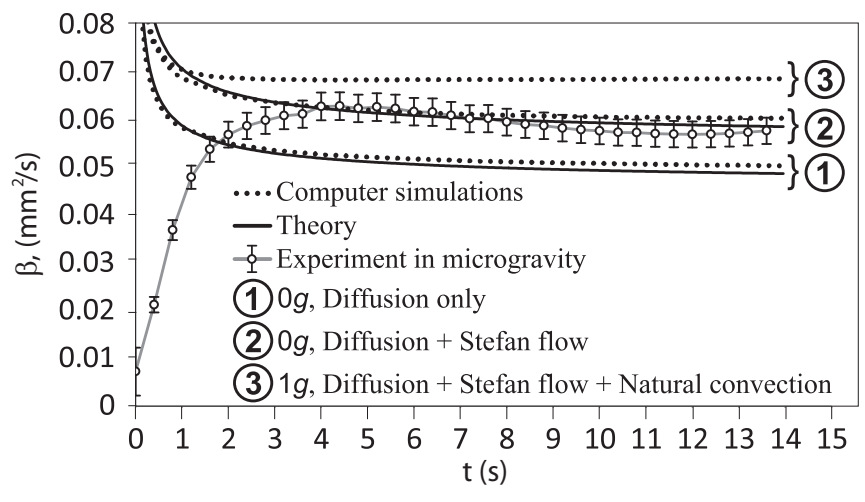

FIG. 4. Normalized evaporation rate $\beta$ from computer simulations, theory [Eqs. (11) and (12)] and experiment in microgravity (the one with $T_{s}-T_{\infty}=15.1 \mathrm{~K}$ ); $0 g$ and $1 g$ stand for zero and terrestrial gravity, respectively.

rate, because the droplet volume changed not only due to evaporation, but also due to pushing liquid back into the injection tubing. This flow was caused by evaporative pressure growth in a closed chamber and, probably, due to the presence of trapped bubbles in the tubing (as a result of aircraft vibrations). This flow resulted in a more intensive change of the droplet's volume than evaporation itself. For this reason, the experimental droplet's evaporation rate has been obtained indirectly with the help of the heat flux meter, as described in the experimental part.

\section{A. Axisymmetric evaporation}

Let us represent the rate of the droplet's volume change as

$$
\frac{d V_{d}}{d t}=-\frac{J(t)}{\rho_{l}}=-\beta L(t) F(\theta(t)) .
$$

Then $\beta=J(t) /\left[\rho_{l} L(t) F(\theta(t))\right]$. It is more practical to compare experimental and theoretical $\beta$ rather than $J$, because in the limit of steady-state evaporation $\left[t \gg L^{2} F^{2}(\theta) /\left(\pi D_{\text {eff }}^{*}\right) \approx\right.$ $0.3 \mathrm{~s}$, that is $t>3 \mathrm{~s}$; see Eq. (11)] $\beta$ weakly depends on changing geometrical parameters $(L$ and $\theta$ ) of the droplet. Note that in general, the contact radius $L(t)$ in Eq. (38) is time dependent. Thus, experimental $\beta$ is computed using experimentally recorded $L(t)$, which basically changes during the initial stage of droplet injection. Theoretical and numerical $\beta$ are computed with constant $L$ according to the models used.

Figure 4 compares normalized evaporation rate $\beta$ from computer simulations, theory, and experiment. Since the contact angle $\theta(t)$ cannot be predicted theoretically, we have used its experimental value, as well as numerically obtained $T_{\mathrm{av}}$ (as mentioned in Sec. II E, $T_{\mathrm{av}}$ is not a fitting parameter), to plot theoretical curves based on Eqs. (11) and (12). One can see that the experimental curve corresponds to the diffusion limited evaporation with Stefan flow (number 2 in Fig. 4). A purely diffusive model (number 1 in Fig. 4) gives a lower evaporation rate, because it does not take into account vapor transport by the Stefan flow, while natural convection under terrestrial gravity enhances drop evaporation (number 3 in Fig. 4).

The discrepancy between experimental and theoretical curves at the process beginning (see number 2 in figure 4 


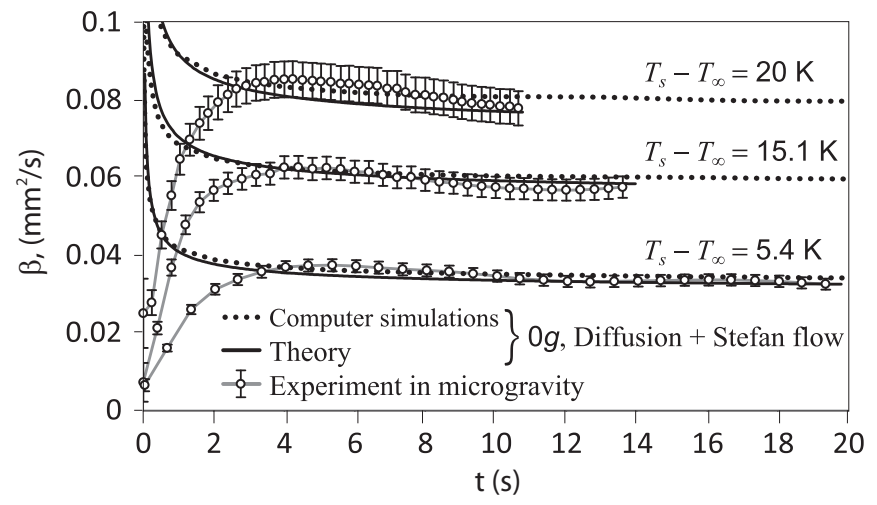

FIG. 5. Normalized evaporation rate $\beta$ for different substrate temperatures.

for $t<3 \mathrm{~s}$ ) is due to different initial conditions. In the experiment there is a stage of droplet injection and spreading (evaporation rate gradually increases with time as droplet grows), while in the theory and computer simulations the contact line is pinned (there is no spreading stage) and evaporation starts instantaneously, resulting in a very high evaporation rate at the beginning. After the first $3 \mathrm{sec}$ (Fig. 4) theory, computer simulations and experiment are in a good quantitative agreement.

Figure 5 compares the normalized evaporation rate $\beta$ for different substrate temperatures. Higher $T_{s}$ gives higher pressure of saturated vapor, and consequently higher evaporation rate. For all given $T_{s}$ at $t>3 \mathrm{~s}$, experimental curves follow the theoretical and numerical ones corresponding to evaporation at zero gravity with Stefan flow taken into account.

The droplets' lifetime is not limited by the experimental points presented in Figs. 4 and 5. Actually, it is much longer and can be easily estimated using the initial droplet volume and steady-state evaporation rate: lifetime $=163,52$, and 48 $\sec$ for $\left(T_{s}-T_{\infty}\right)=5.4,15.1$, and $20 \mathrm{~K}$ respectively. However, experimentally it was impossible to record its full duration due to strict time constraints of the parabolic flight experiments.

Figure 6 demonstrates an effectiveness of the suggested approximation $j_{\text {approx }}$, see Eq. (20), for the local evaporation rate $j$. The expression $\left|j_{\text {approx }}-j\right| / j_{i}^{*}(r, \theta)$ is used to estimate the approximation error. The best fit gives errors less than $10 \%$ for the local flux, whereas global error estimation $\left|J_{\text {approx }}-J\right| / J$ (where $J_{\text {approx }}=\int_{\Gamma} j_{\text {approx }} d \Gamma$ ) shows errors less than $3 \%$. The following fitting parameters have been found for the three particular problems considered in present work: (1) $B=0.4 \mathrm{~K}^{-1}$ and $\Delta h=0.019 \mathrm{~mm}$ for the case $T_{s}-T_{\infty}=5.4 \mathrm{~K}$; (2) $B=0.45 \mathrm{~K}^{-1}$ and $\Delta h=0.019 \mathrm{~mm}$ for the case $T_{s}-T_{\infty}=15.1 \mathrm{~K}$; (3) $B=0.5 \mathrm{~K}^{-1}$ and $\Delta h=0.021$ $\mathrm{mm}$ for the case $T_{s}-T_{\infty}=20 \mathrm{~K}$.

Figure 7 displays computed convective patterns in the gas phase above the droplet. At zero gravity only the Stefan flow is present in the gas phase, which is directed outwards from the droplet surface into the chamber and forms a flow loop near the droplet's contact line. Under terrestrial gravity there are two types of convective vortices in the gas: thermal (due to thermal gas expansion) and solutal (due to nonuniform distribution of vapor, whose density is greater than that of air).

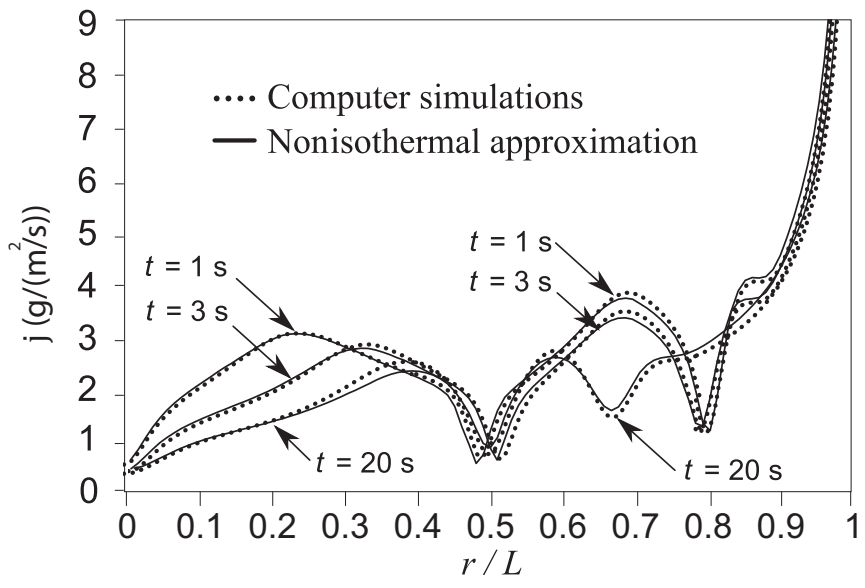

FIG. 6. Fitting local vapor flux $j$ at the liquid-gas interface from our 2D computer simulations (case of $T_{s}-T_{\infty}=15.1 \mathrm{~K}$ ) with approximation $j_{\text {approx }}$ given by Eq. (20). Same parameters $B=0.45 \mathrm{~K}^{-1}$ and $\Delta h=0.019 \mathrm{~mm}$ are used for three presented time moments.

Since in real experiments the droplet injection starts when the gravity level drops down from $2 g$ to $0 g$ (transition from hypergravity to microgravity in a parabolic flight), we have checked numerically the influence of remaining air convection on droplet's evaporation rate. First, a steady-state numerical solution for air convection in the chamber was achieved at $2 g$ gravity level. This solution was used as an initial condition for the next step: droplet evaporation switched on at the moment when the gravity level dropped down to $0 g$.

In this computer simulation with the gravity level change $(2 g \rightarrow 0 g)$, the droplet's evaporation rate did not show any noticeable difference from that obtained numerically in zero gravity. The average gas velocity decreased down to $6 \%$ of its initial value just in $3 \mathrm{sec}$ after the gravity level change, and down to $1 \%$ in 5 sec.

\section{B. Thermoconvective instabilities}

Unsteady computer simulations enabled us to observe thermoconvective instabilities within the drop, see Fig. 8 (multimedia view), which did not appear in the equivalent steady-state problem; see Fig. 9 (time derivatives are neglected in all equations for the steady-state simulation). This result is very important, because it demonstrates a big qualitative difference between unsteady- and steady-state approaches. It shows that steady-state approximation of the problem is not suitable for the study of thermoconvective instabilities. Instead, the full unsteady formulation of the problem must be used.

The unsteady axisymmetric numerical model by Karapetsas et al. [30] also demonstrated the development of a similar thermoconvective pattern in a sessile drop.

In Fig. 8, the initial droplet's temperature coincides with the substrate temperature and equals $315.8 \mathrm{~K}$. The temperature field in the gas phase initially corresponds to the steadystate solution of the heat transport equation (conduction in zero gravity or conduction with buoyancy convection in nonzero gravity) in the gas phase in the absence of droplet 

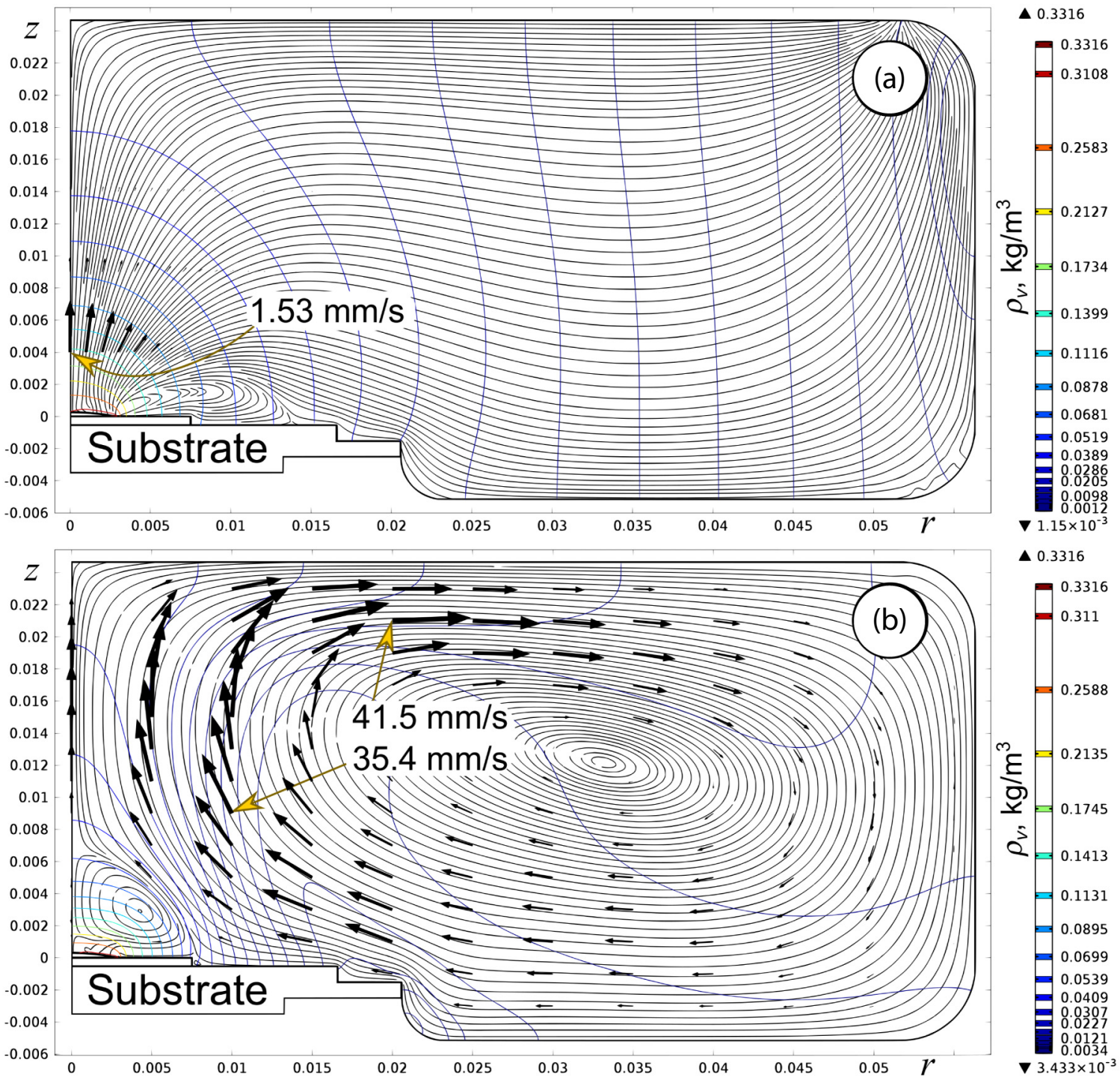

$\Delta 0.3316$

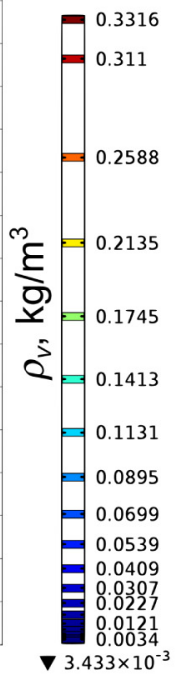

FIG. 7. Computer simulations $\left(T_{s}-T_{\infty}=15.1 \mathrm{~K}, t=14 \mathrm{~s}\right)$ : convective pattern in the gas phase and corresponding contours of vapor density at zero (a) and terrestrial (b) gravity.

evaporation. After evaporation started ( $t=0.001 \mathrm{~s}$ in Fig. 8), the liquid inside of the droplet flows to the three-phase contact line, where local evaporation flux has a maximum value. As evaporation proceeds, the temperature of the liquid-gas interface drops down due to the consumption of the latent heat of vaporization. This temperature change generates temperature gradients along the droplet surface, especially near the contact line, where local evaporation flux is most nonuniform, and the heated substrate is close enough to maintain the high temperature of the contact line. Temperature gradients generate thermocapillary stresses at the interface and drive the liquid flow under the droplet surface: at $t=0.019 \mathrm{~s}$ in Fig. 8 one can see the first thermocapillary vortex propagating from the contact line to the droplet center. As the surface temperature goes down with time, it increases the vertical temperature gradient in the liquid layer, making the system more and more unstable in terms of the Bénard-Marangoni instability. Three subsequent images $(t=0.144 \mathrm{~s}, 0.184 \mathrm{~s}$, and $0.235 \mathrm{~s}$ in Fig. 8) demonstrate a spontaneous development of the Bénard-Marangoni instability: the bottom layers of warmer liquid, being transported to the surface, reinforce the local temperature gradients along the liquid-gas interface and form multiple convective vortices, giving a splash of the velocity magnitude. Further in time, these thermocapillary vortices only slightly change their spatial configuration in response to the changing droplet geometry (contact angle) and remain quasisteady until the end of the computer simulation.

Now let us discuss the type of thermoconvective instability presented in Fig. 8. If the observed instability was due to buoyancy, then we would have substantially different flow patterns at different gravity levels. However, owing to the fact that virtually the same flow pattern, shown in Fig. 8, appears in simulations for both zero gravity and terrestrial gravity, we can conclude that this is definitely not a buoyancy driven convection. Instead, it is driven by tangential thermocapillary stresses at the liquid-gas interface. In general, such flows are subject to different types of thermocapillary instabilities [12]: (a) stationary longitudinal rolls, (b) longitudinal hydrothermal 


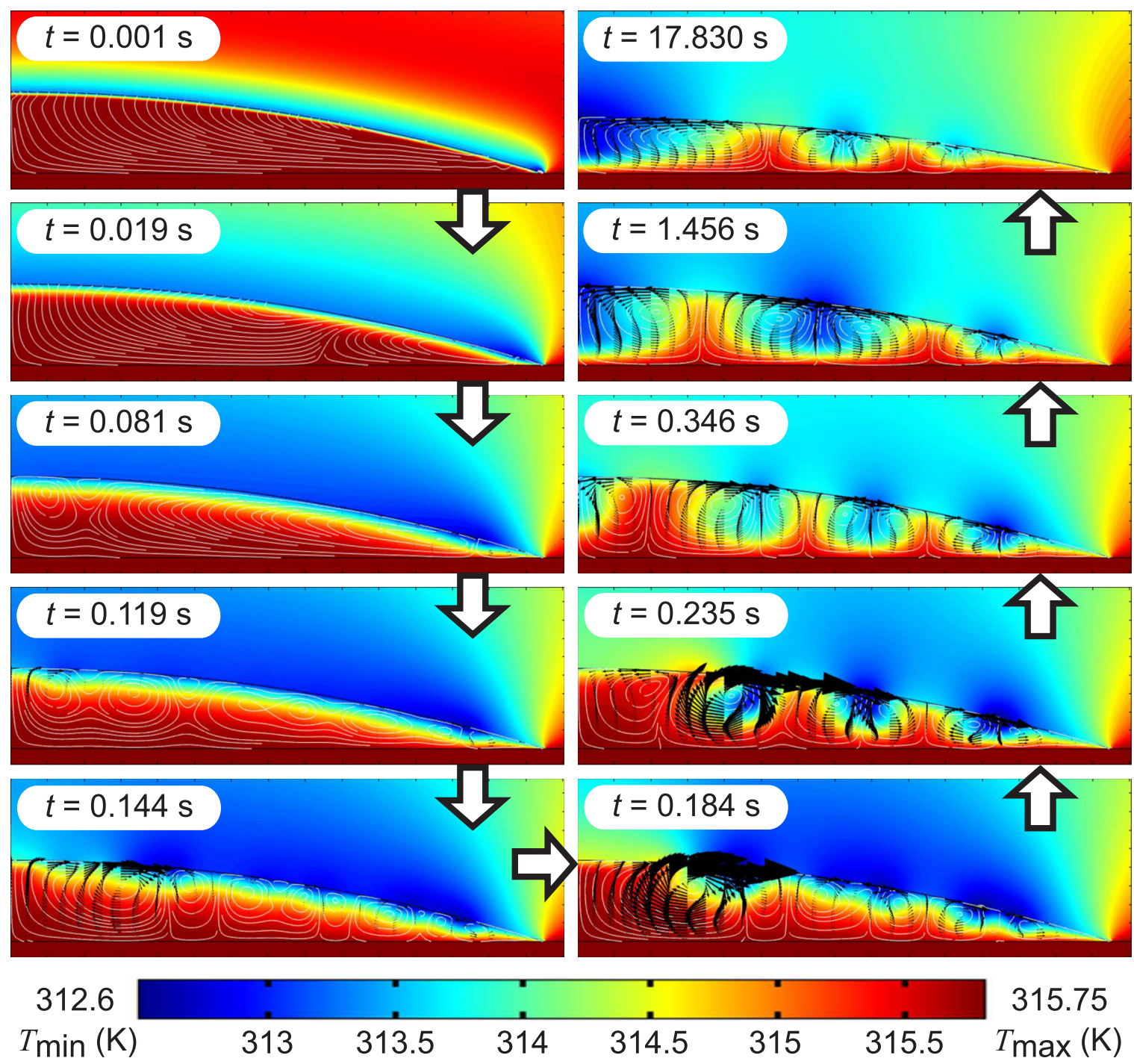

FIG. 8. Unsteady computer simulation: development of thermoconvective instabilities within the drop at $T_{s}-T_{\infty}=15.1 \mathrm{~K}$. Color represents temperature field, white streamlines and black arrows represent velocity in the drop (multimedia view).

waves (HTWs), (c) oblique HTWs, (d) two-dimensional HTWs, and (e) Bénard-Marangoni instability. Instabilities (a)-(c) are essentially three-dimensional phenomena and, therefore, cannot exist in our 2D numerical solution. Type (d) is a secondary unsteady thermoconvective instability, appearing in a basic shear flow in form of waves propagating in the direction of the basic flow [12]. Our numerical solution, see Fig. 8, does not confirm the existence of any basic (underlying observed instability) shear flow in the droplet, or unsteady propagating waves, which proves that these are not HTWs of type (d). Instead, the observed flow structure possesses the features of Bénard-Marangoni instability: steady-state

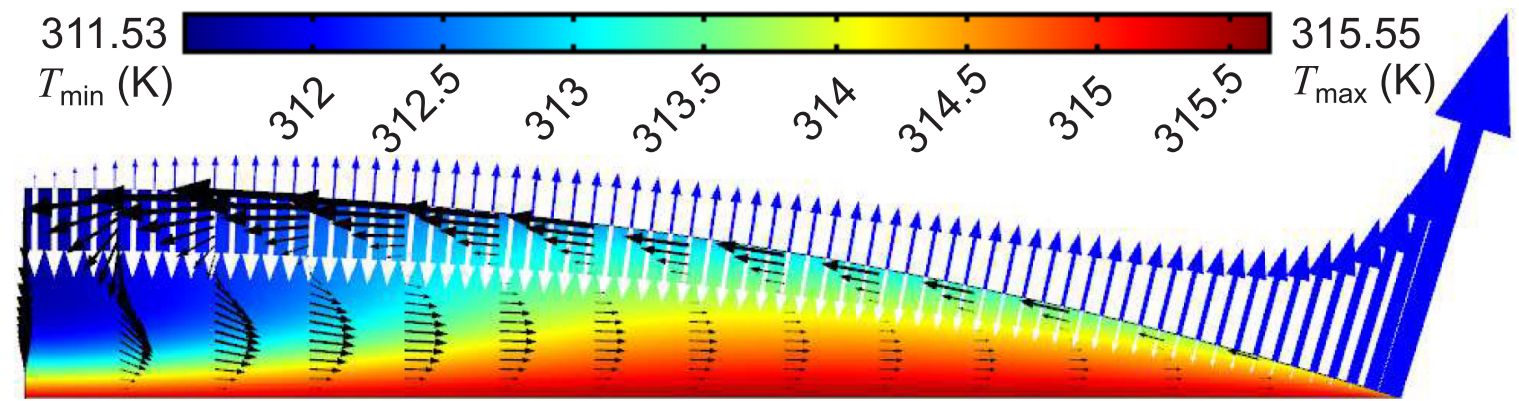

FIG. 9. Steady-state computer simulation: instantaneous distribution of fluxes in the course of droplet evaporation into open atmosphere. Color represents temperature field. Black, white, and blue arrows represent velocity of liquid and interface and evaporation flux, respectively. 
upward liquid flow under hot spots at the droplet surface, and downward flow under cold spots. Thus, we conclude that the numerically obtained instability is the Bénard-Marangoni instability.

For low volatile liquids, our 2D axisymmetric model provides a good agreement between experimental and numerical evaporation rates (see Figs. 4 and 5) meaning that the difference between numerical (2D rolls) and experimental (3D) flow structures has weak influence on the average temperature $T_{\mathrm{av}}$ of the droplet surface, which in turn determines the droplet's evaporation rate [according to Eq. (11)]

\section{CONCLUSIONS}

In this work we have presented theoretical, experimental, and computer simulation results for the evaporation rate of a sessile droplet which are in good quantitative agreement.

A semiempirical theoretical formula has been produced combining a number of exact solutions of simplified problems. It well predicts the unsteady diffusion-limited evaporation rate for a nonisothermal pinned sessile droplet. It takes into account the vapor transport into the gas phase not only by diffusion but also by Stefan flow which is always naturally present and cannot be neglected for intensive evaporation from heated substrates.

A good approximation for the nonisothermal local evaporation flux has been obtained. Its application requires only temperature and tangent velocity at the liquid-gas interface (which are readily available in one-sided numerical models) and contains only two fitting parameters. Thus, this approximation can be used as a boundary condition in one-sided computer simulations of evaporating sessile droplets, especially for 3D problems, when employing two-sided models is computationally expensive.

Using the commercial software COMSOL MULTIPHYSICS, we have developed an unsteady 2D axisymmetric numerical model of nonisothermal evaporation of pinned sessile droplets of ethanol on heated substrates. The fully coupled system of heat and mass transfer equations was solved numerically using arbitrary Lagrangian-Eulerian (ALE) and interface tracking methods to model moving computational mesh which follows the motion of liquid-gas interface.

The unsteady numerical model has demonstrated the formation and development of thermoconvective instabilities in an evaporating sessile droplet. It showed that the steady-state numerical approach is not suitable for the study of these instabilities. Instead, the unsteady numerical approach has to be used.

The current 2D numerical model has demonstrated a good quantitative agreement with the theory and experiments, and combines theoretical, experimental, and computational approaches for the study of unsteady convective evaporation of sessile droplets. In our future work this model is going to be extended into three dimensions, which will allow us to assess the qualitative behavior of 3D thermoconvective instabilities in a sessile drop, and carry out its qualitative validation against experimental observations.

\section{ACKNOWLEDGMENTS}

We would like to acknowledge the financial support of CNES (Centre National d'Études Spatiales) research grant for postdoctorates, ESA (European Space Agency) and the parabolic flight campaigns, as well as Novespace for their technical support. This work has been carried out in the framework of the Labex MEC (ANR-10-LABX-0092) and of the A*MIDEX project (ANR-11-IDEX-0001-02), funded by the Investissements d'Avenir French Government program managed by the French National Research Agency (ANR).

\section{APPENDIX A: DIFFUSION FROM A DISK SOURCE}

Let us show that for zero contact angle $(\theta=0) \mathrm{Eq}$. (13) becomes an exact solution to the problem of steady-state isothermal diffusion-limited evaporation without Stefan flow in the gas phase. In this case our problem is equivalent to diffusion from a disk source with radius $L$. Therefore Eq. (6) from [42] gives us the exact solution for vapor density:

$$
\begin{aligned}
\rho_{v}= & \rho_{v, \infty}+\frac{2}{\pi}\left(\rho_{v, \text { sat }}-\rho_{v, \infty}\right) \\
& \times \arcsin \left(\frac{2 L}{\sqrt{(L-r)^{2}+z^{2}}+\sqrt{(L+r)^{2}+z^{2}}}\right),
\end{aligned}
$$

where $r$ and $z$ are correspondingly radial and vertical cylindrical coordinates. Let us write a Taylor series expansion of (A1) around $z=0$ for $r \leqslant L$ :

$$
\rho_{v}=\rho_{v, \text { sat }}-\frac{2\left(\rho_{v, \text { sat }}-\rho_{v, \infty}\right)}{\pi \sqrt{L^{2}-r^{2}}} z+\mathcal{O}\left(z^{2}\right) .
$$

This allows us to find an exact solution for $j$ :

$$
\begin{aligned}
j(r, 0) & =-\left.D\left(\nabla \rho_{v} \cdot \boldsymbol{n}\right)\right|_{z=0}=-\left.D \frac{\partial \rho_{v}}{\partial z}\right|_{z=0} \\
& =\frac{2 D}{\pi L} \frac{\left(\rho_{v, \mathrm{sat}}-\rho_{v, \infty}\right)}{\sqrt{1-(r / L)^{2}}},
\end{aligned}
$$

where $\boldsymbol{n}$ is the unit vector, normal to the surface of evaporating disk. Thus, for $\theta=0$, approximation (13) becomes the exact solution (A3) when $j_{0}=2 D\left(\rho_{v \text {, sat }}-\rho_{v, \infty}\right) /(\pi L)$.

\section{APPENDIX B: SATURATED VAPOR DENSITY AS A BOUNDARY CONDITION}

Let us justify the use of saturated vapor density as a boundary condition at the liquid-gas interface.

According to the kinetic model of evaporation, vapor density $\left(\rho_{v}\right)_{\mathrm{lg}}$ at the liquid-gas interface can noticeably deviate from the density of saturated vapor, $\rho_{v \text {, sat }}(T)$, if evaporation flux is sufficiently large. In our diffusive model of unsteady evaporation of a sessile droplet into ambient gas, evaporation flux diverges in two different cases: at the beginning of evaporation, when gradient of vapor density is infinitely large at the liquid-gas interface, and at the contact line (singularity), when the contact angle is less than $\pi / 2$. Let us consider these two separate cases below.

In the first case, for simplicity we will consider an isothermal ( $T=\mathrm{const}$ ) and singularity-free (i.e., a sessile 
droplet with contact angle $\theta=\pi / 2$ ) problem. Then, assuming that boundary condition $\left(\rho_{v}\right)_{\mathrm{lg}}=\rho_{v}$, sat holds, we can find the surface density of evaporative mass flux $j$ (which is uniform for $\theta=\pi / 2$ ), across the liquid-gas interface [based on Eq. (11)]:

$$
\begin{aligned}
j & =\frac{J^{\operatorname{Stefan}}(L, T, \pi / 2, t)}{2 \pi L^{2}} \\
& =\frac{D}{L}\left(1+\frac{L}{\sqrt{\pi D t}}\right) \rho_{g} \ln \left(\frac{\rho_{g}-\rho_{v, \infty}}{\rho_{g}-\rho_{v, \text { sat }}}\right) .
\end{aligned}
$$

This solution can be safely used as far as the vapor density at the liquid-gas interface $\left(\rho_{v}\right)_{\lg }$ is close to the saturated one:

$$
\frac{\left|\rho_{v, \text { sat }}-\left(\rho_{v}\right)_{\lg }\right|}{\rho_{v, \text { sat }}} \ll 1 .
$$

Let us estimate the deviation of $\left(\rho_{v}\right)_{\lg }$ from $\rho_{v \text {,sat }}$ using the Hertz-Knudsen-Langmuir equation (evaporation coefficient is assumed to be 1 for simplicity):

$$
j=\sqrt{\frac{R T}{2 \pi M_{e}}}\left[\rho_{v, \mathrm{sat}}-\left(\rho_{v}\right)_{\mathrm{lg}}\right] .
$$

Combining Eqs. (B1) and (B3) we obtain

$$
\begin{aligned}
& \frac{\left|\rho_{v, \text { sat }}-\left(\rho_{v}\right)_{\mathrm{lg}}\right|}{\rho_{v, \text { sat }}} \\
& =\sqrt{\frac{2 \pi M_{e}}{R T}} \frac{D}{L}\left(1+\frac{L}{\sqrt{\pi D t}}\right) \frac{\rho_{g}}{\rho_{v, \text { sat }}}\left|\ln \left(\frac{\rho_{g}-\rho_{v, \infty}}{\rho_{g}-\rho_{v, \text { sat }}}\right)\right| .
\end{aligned}
$$

Let us take $L=3.15 \times 10^{-3} \mathrm{~m}, T=320.8 \mathrm{~K}, p_{\text {atm }}=837$ mbar $=83700 \mathrm{~Pa}, D\left(p_{\mathrm{atm}}, T\right)=1.784 \times 10^{-5} \mathrm{~m}^{2} / \mathrm{s}, M_{e}=$ $0.046 \mathrm{~kg} / \mathrm{mol}, \quad \rho_{v \text {, sat }}(T)=0.456 \mathrm{~kg} / \mathrm{m}^{3}, \quad \rho_{v, \infty}=0 \mathrm{~kg} / \mathrm{m}^{3}$, $\rho_{g}\left(p_{\text {atm }}, T, \rho_{v, \text { sat }}\right)=1.073 \mathrm{~kg} / \mathrm{m}^{3}$. According to Eq. (B4), the difference $\mid \rho_{v}$, sat $-\left(\rho_{v}\right)_{\lg } \mid$ is less than $1 \%$ of $\rho_{v}$, sat for $t>$ $1.06 \times 10^{-5} \mathrm{~s}$.

It means that after the first $10 \mathrm{~ms}$ of evaporation, vapor density above the droplet surface is less than $1 \%$ different from the saturated one. This simple estimation fully justifies the use of saturated vapor density as a boundary condition at the liquid-gas interface in the case of unsteady droplet evaporation.

In the second case, when the contact angle is less than $\pi / 2$, the local evaporation flux diverges at the contact line due to a singularity problem. Let us show that in this case condition (B2) still holds for the most part of the liquid-gas interface even for low contact angles. For example, using the same problem parameters as above and the contact angle $\theta=$ $18.2^{\circ}$, we find that deviation of $\left(\rho_{v}\right)_{\lg }$ from $\rho_{v \text {, sat }}$ is more than $1 \%$ only for a very narrow region next to the contact line. The width of this region can be estimated using Deegan's distribution [see Eq. (6) in [40]] of evaporation flux along the droplet surface, and constitutes only $4.47 \times 10^{-6} \mathrm{~L}$, which is three orders of magnitude less than the size $\left(10^{-2} L\right)$ of a finite element near the contact line. It means that the effect of singularity is absolutely out of the numerical resolution, and can be safely neglected.

Thus, estimating vapor density at the liquid-gas interface (in accordance with the Hertz-Knudsen-Langmuir equation), we have shown that this parameter is very close to the equilibrium one. Therefore, $\rho_{v \text {, sat }}$ can be safely used as a boundary condition at the liquid-gas interface in our theoretical and numerical models.

\section{APPENDIX C: TIME-DEPENDENT DIFFUSION PROBLEM}

The following diffusion equation with the boundary and initial conditions is given to be solved in a spherically symmetric geometry ( $r$ is the radial coordinate):

$$
\left\{\begin{array}{l}
\frac{\partial \rho_{v}}{\partial t}=D \frac{1}{r^{2}} \frac{\partial}{\partial r}\left(r^{2} \frac{\partial \rho_{v}}{\partial r}\right) \\
\left.\rho_{v}\right|_{r=L}=\rho_{v, \text { sat }}\left(T_{\infty}\right),\left.\quad \rho_{v}\right|_{r=\infty}=\rho_{v, \infty},\left.\quad \rho_{v}\right|_{\substack{r>L \\
t=0}}=\rho_{v, \infty}
\end{array} .\right.
$$

Let us introduce new dimensionless variables:

$$
\hat{r}=\frac{r-L}{L}, \quad \hat{t}=\frac{D}{L^{2}} t, \quad \hat{\rho}=\frac{\rho_{v}-\rho_{v, \infty}}{\rho_{v, \text { sat }}\left(T_{\infty}\right)-\rho_{v, \infty}} .
$$

In the new variables, system $(\mathrm{C} 1)$ takes the following form:

$$
\left\{\begin{array}{l}
\frac{\partial \hat{\rho}}{\partial \hat{t}}=\frac{1}{(\hat{r}+1)^{2}} \frac{\partial}{\partial \hat{r}}\left((\hat{r}+1)^{2} \frac{\partial \hat{\rho}}{\partial \hat{r}}\right) \\
\left.\hat{\rho}\right|_{\hat{r}=0}=1,\left.\hat{\rho}\right|_{\hat{r}=\infty}=0,\left.\hat{\rho}\right|_{\substack{\hat{r}>0 \\
\hat{i}=0}}=0
\end{array} .\right.
$$

Without loss of generality we can assume the following form of the solution:

$$
\hat{\rho}(\hat{r}, \hat{t})=\frac{g(\hat{r}, \hat{t})}{\hat{r}+1} .
$$

Then system (C3) becomes

$$
\left\{\begin{array}{l}
\frac{\partial g}{\partial \hat{t}}=\frac{\partial^{2} g}{\partial \hat{r}^{2}} \\
\left.g\right|_{\hat{r}=0} ^{=} 1,\left.g\right|_{\hat{r}=\infty}=g_{\infty}=\text { const, }\left.g\right|_{\substack{\hat{r}>0 \\
\hat{t}=0}}=0
\end{array} .\right.
$$

Now, let us find a self-similarity solution to the problem given by (C5). In short, the self-similarity principle states that if there is such stretching transformation that changes the problem scale but gives the same problem equations and the same boundary and initial conditions, then the solution of this problem should be invariant with respect to such stretching transformation. Let us introduce the following stretching transformation (with positive constant stretching factors $\alpha$, $\tau$, and $\varphi)$ :

$$
r^{*}=\alpha \hat{r}, t^{*}=\tau \hat{t}, g^{*}=\varphi g .
$$

Applying this transformation to the system (C5), we get

$$
\left\{\begin{array}{l}
\frac{\partial g^{*}}{\partial t^{*}}=\frac{\alpha^{2}}{\tau} \frac{\partial^{2} g^{*}}{\partial\left(r^{*}\right)^{2}} \\
\left.g^{*}\right|_{r^{*}=0}=\varphi,\left.g^{*}\right|_{r^{*}=\infty}=\varphi g_{\infty},\left.g^{*}\right|_{\substack{r^{*}>0 \\
t^{*}=0}}=0
\end{array} .\right.
$$

Comparing (C5) and (C7), one can see that problem equations become invariant with respect to this stretching transformation only when

$$
\tau=\alpha^{2} \text { and } \varphi=1 .
$$

The problem solution, $g=g(\hat{r}, \hat{t})$, can be written in the following equivalent implicit form:

$$
F(g, \hat{r}, \hat{t})=0 .
$$

Following the self-similarity principle, the invariance of this solution states that

$$
\begin{array}{rlr}
F(\varphi g, \alpha \hat{r}, \tau \hat{t}) & =0, \text { or taking into account }(\mathrm{C} 8): F\left(g, \alpha \hat{r}, \alpha^{2} \hat{t}\right) \\
& =0 . & (\mathrm{C} 10)
\end{array}
$$


As long as the number of independent variables remains 3, we may change the definition of these variables (by dividing the second argument with the square root of the third argument) to have

$$
F_{1}\left(g, \frac{\hat{r}}{\sqrt{\hat{t}}}, \alpha^{2} \hat{t}\right)=0
$$

Since this functional relation should be true for any value of $\alpha$, it means that dependence of $F_{1}$ on its third argument $\alpha^{2} \hat{t}$ is degenerate, and the solution may take the following form:

$$
F_{2}\left(g, \frac{\hat{r}}{\sqrt{\hat{t}}}\right)=0
$$

which means that the self-similarity solution of problem (C5) should be thought of in the form

$$
g=g(x),
$$

where

$$
x=\frac{\hat{r}}{\sqrt{\hat{t}}}
$$

is the self-similarity variable. Substituting (C13) into the first equation of (C5) and using (C14) we derive an ordinary differential equation for $g$ :

$$
\frac{d^{2} g}{d x^{2}}+\frac{x}{2} \frac{d g}{d x}=0 .
$$

The general solution of Eq. (C15) is given by

$$
g=C_{1}+C_{2} \operatorname{erf}\left(\frac{x}{2}\right)=C_{1}+C_{2} \operatorname{erf}\left(\frac{\hat{r}}{\sqrt{4 \hat{t}}}\right),
$$

where $C_{1}$ and $C_{2}$ are some constants, and $\operatorname{erf}(x)$ is the Gaussian error function:

$$
\operatorname{erf}(x)=\frac{2}{\sqrt{\pi}} \int_{0}^{x} e^{-t^{2}} d t .
$$

Applying the boundary and initial conditions from (C5) to the solution (C16) gives

$$
C_{1}=1, C_{2}=-1, \text { and } g_{\infty}=0,
$$

resulting in the following problem solution:

$$
g=\operatorname{erfc}\left(\frac{\hat{r}}{\sqrt{4 \hat{t}}}\right)
$$

where $\operatorname{erfc}(x)=1-\operatorname{erf}(x)$ is a complementary error function. Finally, combining (C19) with (C4) and (C2), we obtain the problem solution:

$$
\rho_{v}=\rho_{v, \infty}+\left[\rho_{v, \text { sat }}\left(T_{\infty}\right)-\rho_{v, \infty}\right] \frac{L}{r} \operatorname{erfc}\left(\frac{r-L}{\sqrt{4 D t}}\right) .
$$

Using (C20) one can find the surface density of the diffusive vapor mass flux at the droplet surface:

$$
\begin{aligned}
j^{\operatorname{dif}}\left(L, T_{\infty}, t\right)= & -\left.D \frac{\partial \rho_{v}}{\partial r}\right|_{r=L}=D\left[\rho_{v, \text { sat }}\left(T_{\infty}\right)-\rho_{v, \infty}\right] \\
& \times \frac{1}{L}\left(1+\frac{L}{\sqrt{\pi D t}}\right),
\end{aligned}
$$

which leads to the following total evaporation rate of a spherical droplet:

$$
\begin{aligned}
J_{\mathrm{sph}}^{\mathrm{dif}}\left(L, T_{\infty}, t\right)= & 4 \pi L^{2} j^{\mathrm{dif}}\left(L, T_{\infty}, t\right)=4 \pi D L\left[\rho_{v, \text { sat }}\left(T_{\infty}\right)\right. \\
& \left.-\rho_{v, \infty}\right]\left(1+\frac{L}{\sqrt{\pi D t}}\right) .
\end{aligned}
$$

The last equation is identical to Eq. (9).

\section{APPENDIX D: JUSTIFICATION OF FORMULA (19)}

Let us justify formula (19) by deriving it from Eq. (18). In the right-hand side of Eq. (18), the integrand is nothing more than a triple product, which allows cyclic permutation of vectors:

$$
\boldsymbol{j}_{\tau} \cdot[d \boldsymbol{l} \times \boldsymbol{n}]=\left(\boldsymbol{j}_{\tau}, d \boldsymbol{l}, \boldsymbol{n}\right)=\left(d \boldsymbol{l}, \boldsymbol{n}, \boldsymbol{j}_{\tau}\right)=d l \cdot\left[n \times \boldsymbol{j}_{\tau}\right] .
$$

Next, applying the curl theorem to the right-hand side of Eq. (18), we obtain

$$
\oint_{\partial S} \boldsymbol{d l} \cdot\left[\boldsymbol{n} \times \boldsymbol{j}_{\tau}\right]=\iint_{S}\left[\nabla \times\left[\boldsymbol{n} \times \boldsymbol{j}_{\tau}\right]\right] \cdot \boldsymbol{n} d S,
$$

where $\nabla$ is the nabla operator. Comparing Eqs. (18) and (D2) we derive

$$
j_{+}=\Delta h\left[\nabla \times\left[\boldsymbol{n} \times \boldsymbol{j}_{\tau}\right]\right] \cdot \boldsymbol{n} .
$$

For simplicity we will further develop this expression in a three-dimensional Cartesian system of coordinates $x^{i}(i=1,2,3)$ with constant covariant $\boldsymbol{e}_{i}$ and contravariant $\boldsymbol{e}^{i}$ basis vectors of unit length. Then, any vector $\boldsymbol{v}$ can be written as

$$
\boldsymbol{v}=v^{i} \boldsymbol{e}_{i}=\left(\boldsymbol{v} \cdot \boldsymbol{e}^{i}\right) \boldsymbol{e}_{i},
$$

where the Einstein summation convention for repeated indices is used. If vector $\boldsymbol{v}$ represents a vector product of two vectors $\boldsymbol{a}$ and $\boldsymbol{b}$, then we have

$$
\begin{aligned}
{[\boldsymbol{a} \times \boldsymbol{b}] } & =\boldsymbol{v}=\left(\boldsymbol{v} \cdot \boldsymbol{e}^{i}\right) \boldsymbol{e}_{i}=\left([\boldsymbol{a} \times \boldsymbol{b}] \cdot \boldsymbol{e}^{i}\right) \boldsymbol{e}_{i}=\left(\left[a^{k} \boldsymbol{e}_{k} \times b^{l} \boldsymbol{e}_{l}\right] \cdot \boldsymbol{e}^{i}\right) \boldsymbol{e}_{i} \\
& =a^{k} b^{l} \underbrace{\left[\left[\boldsymbol{e}_{k} \times \boldsymbol{e}_{l}\right] \cdot \boldsymbol{e}^{i}\right)}_{\varepsilon_{k l}{ }^{i}} \boldsymbol{e}_{i}=a^{k} b^{l} \varepsilon_{k l}{ }^{i} \boldsymbol{e}_{i}, \quad \text { (D5) }
\end{aligned}
$$

where $\varepsilon_{k l}^{i}=\left(\boldsymbol{e}_{k}, \boldsymbol{e}_{l}, \boldsymbol{e}^{i}\right)$ are the components of the Levi-Civita tensor. It is now more convenient to write expression (D3) in an index notation:

$$
\frac{j_{+}}{\Delta h}=\varepsilon_{i m}^{q} \partial_{q}\left(n^{k} j_{\tau}^{l} \varepsilon_{k l}^{i}\right) n^{m}
$$

where $\partial_{q}$ stands for the partial derivative $\frac{\partial}{\partial x^{q}}$. Since basis vectors $\boldsymbol{e}_{i}$ or $\boldsymbol{e}^{i}$, and subsequently the components of LeviCivita tensor, are constants, then the symbol $\varepsilon_{k l}{ }^{i}$ in Eq. (D6) can be taken outside the derivative:

$$
\frac{j_{+}}{\Delta h}=\varepsilon_{i m}^{q} \varepsilon_{k l}^{i} \partial_{q}\left(n^{k} j_{\tau}^{l}\right) n^{m}
$$


Now we can apply the following relation between the product of the Levi-Civita tensor components, $\varepsilon_{i m}^{q} \varepsilon_{k l}{ }^{i}$, the components of the metric tensor, $g_{i k}=\boldsymbol{e}_{i} \cdot \boldsymbol{e}_{k}$ or $g^{i k}=\boldsymbol{e}^{i} \cdot \boldsymbol{e}^{k}$, and the Kronecker delta symbols $\delta_{i}^{k}=\boldsymbol{e}_{i} \cdot \boldsymbol{e}^{k}$ :

$$
\varepsilon_{i m}^{q} \varepsilon_{k l}^{i}=\left|\begin{array}{ccc}
\delta_{k}^{q} & \delta_{l}^{q} & g^{q i} \\
g_{i k} & g_{i l} & \delta_{i}^{i} \\
g_{m k} & g_{m l} & \delta_{m}^{i}
\end{array}\right|=\delta_{l}^{q} g_{m k}-\delta_{k}^{q} g_{m l} .
$$

Substituting (D8) into (D7), we obtain

$$
\begin{aligned}
\frac{j_{+}}{\Delta h} & =\left(\delta_{l}^{q} g_{m k}-\delta_{k}^{q} g_{m l}\right) \partial_{q}\left(n^{k} j_{\tau}^{l}\right) n^{m} \\
& =\partial_{l}\left(n^{k} j_{\tau}^{l}\right) n_{k}-\partial_{k}\left(n^{k} j_{\tau}^{l}\right) n_{l},
\end{aligned}
$$

or after expanding derivatives

$$
\frac{j_{+}}{\Delta h}=\underbrace{n_{k} \partial_{l}\left(n^{k}\right)}_{=0} j_{\tau}^{l}+\partial_{l}\left(j_{\tau}^{l}\right) \underbrace{n^{k} n_{k}}_{=1}-\partial_{k}\left(n^{k}\right) \underbrace{j_{\tau}^{l} n_{l}}_{=0}-\partial_{k}\left(j_{\tau}^{l}\right) n^{k} n_{l} .
$$

Note that, since normal vector $\boldsymbol{n}$ has fixed unit length $\left[n_{k} n^{k}=1\right.$ and hence $\left.2 n_{k} \partial_{l}\left(n^{k}\right)=\partial_{l}\left(n_{k} n^{k}\right)=0\right]$, and the tangential vapor flux $\boldsymbol{j}_{\tau}$ is orthogonal to the normal vector $\left(\boldsymbol{j}_{\tau} \cdot \boldsymbol{n}=j_{\tau}{ }^{l} n_{l}=0\right)$, expression (D10) is simplified:

$$
\frac{j_{+}}{\Delta h}=\partial_{l}\left(j_{\tau}^{l}\right)-n^{k} n_{l} \partial_{k}\left(j_{\tau}^{l}\right)=\left(\partial_{l}-n^{k} n_{l} \partial_{k}\right) j_{\tau}^{l},
$$

which in vector notation corresponds to

$$
j_{+}=\Delta h \underbrace{(\nabla-(\boldsymbol{n} \otimes \boldsymbol{n}) \cdot \nabla)}_{\nabla_{\Gamma}} \cdot \boldsymbol{j}_{\tau}=\Delta h \nabla_{\Gamma} \cdot \boldsymbol{j}_{\tau},
$$

which is identical to Eq. (19).
[1] P. Boulet, J. Tissot, F. Trinquet, and L. Fournaison, Appl Thermal Eng. 50, 1164 (2013).

[2] J. Chen, W.-S. Liao, X. Chen, T. Yang, S. E. Wark, D. H. Son, J. D. Batteas, and P. S. Cremer, ACS Nano 3, 173 (2009).

[3] F. Carle and D. Brutin, Langmuir 29, 9962 (2013).

[4] J. H. Combellack, Crop Prot. 3, 9 (1984).

[5] S. H. Ko, J. Chung, N. Hotz, K. H. Nam, and C. P. Grigoropoulos, J. Micromech. Microeng. 20, 125010 (2010).

[6] N. C. Schirmer, S. Ströhle, M. K. Tiwari, and D. Poulikakos, Adv. Funct. Mater. 20, 1 (2010).

[7] R. Danzebrink and M. A. Aegerter, Thin Solid Films 392, 223 (2001).

[8] P. G. Campbell and L. E. Weiss, Expert Opin. Biol. Ther. 7, 1123 (2007).

[9] W. Bou-Zeid and D. Brutin, Colloid Surf. A 456, 273 (2014).

[10] B. Sobac and D. Brutin, Colloid Surf. A 448, 34 (2014).

[11] D. Halpern, H. Fujioka, S. Takayama, and J. B. Grotberg, Respir Physiol. Neuro. 163, 222 (2008).

[12] M. K. Smith and S. H. Davis, J. Fluid Mech. 132, 119 (1983).

[13] M. K. Smith and S. H. Davis, J. Fluid Mech. 132, 145 (1983).

[14] N. Garnier, A. Chiffaudel, and F. Daviaud, in Dynamics of Spatio-temporal Cellular Structures (Springer, Berlin, New York, 2006), pp. 147-161.

[15] P. J. Saenz, P. Valluri, K. Sefiane, G. Karapetsas, and O. K. Matar, Phys. Fluids 25, 094101 (2013).

[16] D. Schwabe, A. Zebib, and B.-C. Sim, J. Fluid Mech. 491, 239 (2003).

[17] K. Sefiane, J. R. Moffat, O. K. Matar, and R. V. Craster, Appl. Phys. Lett. 93, 074103 (2008).

[18] K. Sefiane, Y. Fukatani, Y. Takata, and J. Kim, Langmuir 29, 9750 (2013).

[19] F. Carle, B. Sobac, and D. Brutin, J. Fluid Mech. 712, 614 (2012).

[20] D. Brutin, B. Sobac, F. Rigollet, and C. L. Niliot, Exp. Therm. Fluid Sci. 35, 521 (2011).

[21] B. Sobac and D. Brutin, Phys. Fluids 24, 032103 (2012).

[22] L. Y. Barash, T. P. Bigioni, V. M. Vinokur, and L. N. Shchur, Phys. Rev. E 79, 046301 (2009).

[23] K. L. Maki and S. Kumar, Langmuir 27, 11347 (2011).
[24] A. E. Korenchenko and V. P. Beskachko, Phys. Fluids 25, 112106 (2013).

[25] K. Yang, F. Hong, and P. Cheng, Int. J. Heat Mass Transfer 70, 409 (2014).

[26] M. Gross, F. Varnik, D. Raabe, and I. Steinbach, Phys. Rev. E 81, 051606 (2010).

[27] F. Varnik, M. Gross, N. Moradi, G. Zikos, P. Uhlmann, P. MüllerBuschbaum, D. Magerl, D. Raabe, I. Steinbach, and M. Stamm, J. Phys.: Condens. Matter 23, 184112 (2011).

[28] J. B. Miller, A. C. P. Usselman, R. J. Anthony, U. R. Kortshagen, A. J. Wagner, A. R. Denton, and E. K. Hobbie, Soft Matter 10, 1665 (2014).

[29] C. Xie, J. Zhang, V. Bertola, and M. Wang, J. Colloid Interf. Sci. 463, 317 (2016).

[30] G. Karapetsas, O. K. Matar, P. Valluri, and K. Sefiane, Langmuir 28, 11433 (2012).

[31] F. Carle, S. Semenov, M. Medale, and D. Brutin, Int. J. Therm. Sci. 101, 35 (2016).

[32] B. Sobac, P. Talbot, B. Haut, A. Rednikov, and P. Colinet, J. Colloid Interface Sci. 438, 306 (2015).

[33] D. W. Green and R. H. Perry, Perry's Chemical Engineers' Handbook, 8th ed. (McGraw-Hill, New York, 2007).

[34] R. C. Reid and T. K. Sherwood, The Properties of Gases and Liquids (McGraw-Hill, New York, 1958).

[35] D. B. Spalding, in Proceedings of the 4th International Symposium on Combustion (Williams and Wilkins, Baltimore, 1953), Vol. 4, pp. 847-864.

[36] R. G. Picknett and R. Bexon, J. Colloid Interface Sci. 61, 336 (1977).

[37] S. Semenov, V. Starov, and R. G. Rubio, Droplet Wetting and Evaporation (Academic, New York, 2015), pp. 315-337.

[38] Y. O. Popov, Phys. Rev. E 71, 036313 (2005).

[39] S. Semenov, V. M. Starov, R. G. Rubio, and M. G. Velarde, Colloid Surf. A 372, 127 (2010).

[40] R. D. Deegan, O. Bakajin, T. F. Dupont, G. Huber, S. R. Nagel, and T. A. Witten, Phys. Rev. E 62, 756 (2000).

[41] S. Semenov, V. M. Starov, and R. G. Rubio, Eur. Phys. J. Spec. Top. 219, 143 (2013).

[42] A. W. Warrick, P. Broadbridge, and D. O. Lomen, Appl. Math. Model. 16, 155 (1992). 